To László Babai: for asking questions that keep us very entertained.

\title{
CLASSIFICATION OF FINITE GROUPS THAT ADMIT AN ORIENTED REGULAR REPRESENTATION
}

\author{
JOY MORRIS AND PABLO SPIGA
}

\begin{abstract}
This is the third, and last, of a series of papers dealing with oriented regular representations. Here we complete the classification of finite groups that admit an oriented regular representation (or ORR for short), and give a complete answer to a 1980 question of László Babai: "Which [finite] groups admit an oriented graph as a DRR?" It is easy to see and wellunderstood that generalised dihedral groups do not admit ORRs. We prove that, with 11 small exceptions (having orders ranging from 8 to 64), every finite group that is not generalised dihedral has an ORR.
\end{abstract}

\section{INTRODUCTION}

All groups and graphs in this paper are finite. Let $G$ be a group and let $S$ be a subset of $G$. The Cayley digraph, denoted by Cay $(G, S)$, over $G$ with connection set $S$ is the digraph with vertex set $G$ and with $(x, y)$ being an arc if $y x^{-1} \in S$. (An arc is an ordered pair of adjacent vertices.) Since the group $G$ acts faithfully as a group of automorphisms of $\operatorname{Cay}(G, S)$ via the right regular representation, Cayley digraphs represent groups geometrically and combinatorially as groups of automorphisms of digraphs. Naively, the closer $G$ is to the full automorphism group of Cay $(G, S)$, the closer this representation is to a precise graphical encoding of $G$.

Following this line of thought, it is natural to ask which groups $G$ admit a subset $S$ with $G$ being the automorphism group of $\operatorname{Cay}(G, S)$; that is, $\operatorname{Aut}(\operatorname{Cay}(G, S))=$ $G$. We say that $G$ admits a digraphical regular representation (or DRR for short) if there exists a subset $S$ of $G$ with $\operatorname{Aut}(\operatorname{Cay}(G, S))=G$. Babai [1, Theorem 2.1] has given a complete classification of the groups admitting a DRR: except for

$$
Q_{8}, C_{2}^{2}, C_{2}^{3}, C_{2}^{4} \text { and } C_{3}^{2} \text {, }
$$

every group admits a DRR.

In light of Babai's result, it is natural to try to combinatorially represent groups as automorphism groups of special classes of Cayley digraphs. Observe that, if $S$ is inverse-closed (that is, $S=S^{-1}:=\left\{s^{-1} \mid s \in S\right\}$ ), then Cay $(G, S)$ is undirected. Now, we say that $G$ admits a graphical regular representation (or GRR for short) if there exists an inverse-closed subset $S$ of $G$ with $\operatorname{Aut}(\operatorname{Cay}(G, S))=G$. With a considerable amount of work culminating in [6, 8, the groups admitting a GRR have been completely classified. (In fact, this question attracted significant interest well before the DRR problem, although the final solutions to both problems appeared at about the same time.)

We recall that a tournament is a digraph $\Gamma:=(V, A)$ with vertex set $V$ and arc set $A$ such that, for every two distinct vertices $x, y \in V$, exactly one of $(x, y)$ and

This research was supported in part by the National Science and Engineering Research Council of Canada grant RGPIN-2017-04905. 
$(y, x)$ is in $A$. After the completion of the classification of DRRs and GRRs, Babai and Imrich [2] proved that every group of odd order except for $C_{3}^{2}$ and $C_{3}^{3}$ admits a tournament regular representation (or TRR for short). That is, each finite odd-order group $G$ different from $C_{3}^{2}$ and $C_{3}^{3}$ contains a subset $S$ with Cay $(G, S)$ being a tournament and with $\operatorname{Aut}(\operatorname{Cay}(G, S))=G$. In terms of the connection set $S$, the Cayley digraph $\operatorname{Cay}(G, S)$ is a tournament if and only if $S \cap S^{-1}=\emptyset$ and $G \backslash\{1\}=S \cup S^{-1}$. This observation makes it clear that a Cayley digraph on $G$ cannot be a tournament if $G$ contains an element of order 2 , so only groups of odd order can admit TRRs.

In [1, Problem 2.7], Babai observed that one class of Cayley digraphs is rather interesting and had not been investigated in the context of regular representations; that is, the class of oriented Cayley digraphs (or as Babai called them, oriented Cayley graphs). An oriented Cayley digraph is in some sense a "proper" Cayley digraph. More formally, it is a Cayley digraph $\operatorname{Cay}(G, S)$ whose connection set $S$ has the property that $S \cap S^{-1}=\emptyset$. Equivalently, in graph-theoretic terms, it is a Cayley digraph with no digons.

Definition 1.1. The group $G$ admits an oriented regular representation (or ORR for short) if there exists a subset $S$ of $G$ with $S \cap S^{-1}=\emptyset$ and $\operatorname{Aut}(\operatorname{Cay}(G, S))=$ $G$.

Babai asked in [1 which (finite) groups admit an ORR. Since a TRR is a special type of ORR, and $C_{3}^{2}$ is one of the five groups in Eq. 1 that do not admit a DRR (so cannot admit an ORR), the answer to this question for groups of odd order was already known when Babai published his question.

In this paper, answering the question of Babai and also confirming the conjecture given in [12, Conjecture 1.5], we prove the following result.

Theorem 1.2. Every finite group $G$ admits an ORR, unless one of the following holds:

(i): $G$ is generalised dihedral with $|G|>2$ (see Definition 2.1 for the meaning of generalised dihedral);

(ii): $G$ is isomorphic to one of the following eleven groups

$$
\begin{aligned}
& Q_{8}, C_{4} \times C_{2}, C_{4} \times C_{2}^{2}, C_{4} \times C_{2}^{3}, C_{4} \times C_{2}^{4}, C_{3}^{2}, C_{3} \times C_{2}^{3}, \\
& \left.\left\langle a, b \mid a^{4}=b^{4}=(a b)^{2}=\left(a b^{-1}\right)^{2}=1\right\rangle \text { (of order } 16\right), \\
& \langle a, b, c| a^{4}=b^{4}=c^{4}=(b a)^{2}=\left(b a^{-1}\right)^{2}=(b c)^{2}=\left(b c^{-1}\right)^{2}=1, \\
& \left.\left.\qquad a^{2}=c^{2}, a^{c}=a^{-1}, a^{2}=b^{2}\right\rangle \text { (of order } 16\right), \\
& \langle a, b, c| a^{4}=b^{4}=c^{4}=(a b)^{2}=\left(a b^{-1}\right)^{2}=1, \\
& \left.\left.\quad(a c)^{2}=\left(a c^{-1}\right)^{2}=(b c)^{2}=\left(b c^{-1}\right)^{2}=a^{2} b^{2} c^{2}=1\right\rangle \text { (of order } 32\right), \\
& D_{4} \circ D_{4} \quad \text { (the central product of two dihedral groups of order } 8, \\
& \quad \text { which is the extraspecial group of order } 32 \text { of plus type). }
\end{aligned}
$$

We remark that since this theorem relies on results in the previous two papers [12, 13] and since the results in [12] depend upon the Classification of Finite Simple Groups, this theorem also depends on the Classification.

In our opinion this is not the final word on oriented regular representations of finite groups. In fact, it is still unclear whether ORRs behave asymptotically like DRRs and GRRs. (It is believed that most Cayley digraphs are DRRs and that 
most Cayley graphs are GRRs. One should be very careful about how to understand "most" in these statements and we refer the reader to the introduction of [5] for two distinct, natural interpretations of "most".)

We conclude this introductory section by observing that regular representations have shown a new vitality lately. For instance, Marston Conder, Mark Watkins and Tom Tucker [4 have been studying finite groups admitting a graphical Frobenius representation and have posed some very intriguing conjectures in this context. All of these conjectures are in line with the classification of groups that admit DRRs, GRRs, TRRs and now ORRs: except for some "low level noise" (yielding a finite number of small exceptions) and any obvious obstructions, regular representations of the desired type will exist. For DRRs, there are no obvious general obstructions; for TRRs, groups of even order are problematic and yield the only general obstruction; for GRRs, groups admitting automorphisms that map each element to itself or to its inverse are problematic and yield the only general obstruction; for ORRs, groups for which every generating set contains at least one involution (that is, generalised dihedral groups) are problematic and (in light of Theorem 1.2) yield the only general obstruction.

Finally, we refer to $5,11,14,15$ for some recent work on similar problems.

\section{EARLIER WORK AND PRELIMINARIES}

Before moving to the proof of Theorem 1.2 we need to review the main results that have been proved on oriented regular representations. We start with a few definitions.

Babai pointed out in [1] that generalised dihedral groups of order greater than 2 can never admit an ORR. (Given a group element $g$, we denote by $o(g)$ its order.)

Definition 2.1. Let $A$ be an abelian group. The generalised dihedral group over $A$ is the group $\langle\tau, A\rangle$ with $o(\tau)=2$ and $\tau a \tau=a^{-1}$ for every $a \in A$.

In the special case where $A$ is cyclic, this is the dihedral group over $A$. Observe that, unless $|G|=2$, if $\operatorname{Cay}(G, S)$ is an $\operatorname{ORR}$, then $\operatorname{Cay}(G, S)$ is connected and hence $S$ is a generating set for $G$. Now, Babai's observation follows immediately from the fact that if $G$ is the generalised dihedral group over the abelian group $A$, then every element of $G \backslash A$ has order 2. Thus every generating set $S$ for $G$ must contain an involution, so that $S \cap S^{-1} \neq \emptyset$. This renders understanding generalised dihedral groups very important when we are studying ORRs.

Let $G$ be a finite group. As customary, we denote by $d(G)$ the minimum number of generators for $G$. Following [13, Section 2], we say that a generating set $\left\{g_{1}, \ldots, g_{d}\right\}$ for $G$ is irredundant if, for each $i \in\{1, \ldots, d\}$, the $d-1$ elements

$$
g_{1}, g_{2}, \ldots, g_{i-1}, g_{i+1}, \ldots, g_{d}
$$

do not generate $G$. Observe that each generating set for $G$ of cardinality $d(G)$ is irredundant.

We say that the $d$-tuple $\left(g_{1}, \ldots, g_{d}\right)$ of elements of $G$ is beautiful if the following conditions hold:

(i): $\left\{g_{1}, \ldots, g_{d}\right\}$ is an irredundant generating set for $G$,

(ii): $o\left(g_{i}\right)>2$ for every $i \in\{1, \ldots, d\}$,

(iii): $o\left(g_{i+1} g_{i}^{-1}\right)>2$ for every $i \in\{1, \ldots, d-1\}$. 
Observe that being beautiful is a property of ordered tuples and not of sets; that is, it depends upon the ordering of the generating set $\left\{g_{1}, \ldots, g_{d}\right\}$ for $G$.

An important connection between beautiful generating tuples and ORRs is given in the next theorem.

Theorem 2.2. Let $G$ be a finite group admitting a beautiful generating tuple. Then $G$ admits an ORR if and only if $G \neq Q_{8}, G \neq C_{3} \times C_{2}^{3}$, and $G \neq C_{3} \times C_{3}$.

This theorem is implicit in [12] and follows immediately from the theory developed therein. For a proof see [13, Theorem 2.1].

Developing the theory of beautiful generating tuples (and actually something more general, which we called five-product-avoiding generating sets), we have proved in 12 that each non-soluble group admits an ORR. Building on this result, the second author has proved the following result, which (among other things) reduces the classification of groups admitting an ORR to some very specific infinite families of 2-groups.

Theorem 2.3 (([13], Theorem 1.2)). Let $G$ be a finite group. Then one of the following holds:

(i): $G$ admits an ORR;

(ii): $G$ has an abelian 2-subgroup $A$, a normal subgroup $N$ and two elements $g \in G \backslash N$ and $n \in N \backslash A$ with $A<N<G,|G: N|=|N: A|=2, g^{2}=1$, $n^{g}=n^{-1}$ and $a^{g}=a^{-1}$ for each $a \in A$;

(iii): there exists a normal subgroup $N$ of $G, g \in G$ and $n_{0} \in N$ with $|G: N|=$ $2, G=\langle N, g\rangle, g^{2}=1, N$ is a 2-group and the action of $g$ by conjugation on $N$ inverts precisely half of the elements of $N$ and $N=H \cup n_{0} H$, where $H:=\left\{n \in N \mid n^{g}=n^{-1}\right\}$. Moreover, $N$ has no automorphism inverting more than half of its elements. (Every group $N$ that has an automorphism inverting half of its elements and no automorphism that inverts more is classified in [7] by Hegarty and MacHale);

(iv): $G$ is isomorphic to $Q_{8}$, to $C_{3} \times C_{3}$ or to $C_{3} \times C_{2}^{3}$;

(v): $G$ is generalised dihedral.

In view of this theorem, the classification of finite groups admitting an ORR is reduced to the groups in (ii) and (iii). We address these two families in Sections 4 and 5 , respectively.

We remark that the "flavour" of many of the ORRs that are produced in this paper is quite different from those produced in [12] and [13. Those papers focused on the use of beautiful generating tuples, so that for any two consecutive elements $g_{i}$ and $g_{i+1}$ of the generating tuple, the product $g_{i+1} g_{i}^{-1}$ has order greater than 2 . As we will see, a common situation for a group $G$ in the families we study in this paper is that $G$ contains an elementary abelian subgroup $B$ of high rank and low index. In order to generate such a group $G$, we require a large number of elements from at least one of the cosets $B g$ of $B$. If we place any two elements $g_{i}:=b_{1} g$ and $g_{i+1}:=b_{2} g$ of $B g$ (where $b_{1}, b_{2} \in B$ ) consecutively in the generating set, then $g_{i+1} g_{i}^{-1}=b_{2} g g^{-1} b_{1}^{-1}=b_{2} b_{1}^{-1} \in B$, so $o\left(g_{i+1} g_{i}^{-1}\right) \leq 2$. In principle it could be possible to ensure that whenever $g_{i} \in B g$ we have $g_{i+1} \notin B g$, but in practice we may still find that $o\left(g_{i+1} g_{i}^{-1}\right)=2$. We will therefore take a very different approach that uses a GRR for $B$ as a starting point. In our previous papers, if we look at the induced subdigraph of each of our ORRs on the vertices that lie in the connection set (equivalently, the induced subdigraph on the open neighbourhood 
of any vertex), that digraph is (weakly) connected and asymmetric. The ORRs we produce in this paper will often include many isolated vertices in that induced subdigraph.

Before moving into our analysis of the groups in (ii) and (iii) (from above), we introduce some other results from the literature that will be important in our proofs.

The following result is found in the proof of the theorem in 9. Note that the statement in [9] assumes only $k \geq 5$, but there is a mistake in the case $k=5$ that has been pointed out by multiple researchers. Although the elementary abelian 2 -group of rank 5 also admits a GRR, it requires a different connection set so we omit it from our statement.

Lemma 2.4 ((Imrich [9])). Let $G$ be an elementary abelian 2-group of rank $k \geq 6$, and let $\left\{x_{1}, \ldots, x_{k}\right\}$ be a generating set for $G$. Then $G$ has a GRR; furthermore, a GRR is given by the Cayley graph on $G$ whose connection set consists of the $2 k+1$ elements:

$$
x_{1}, \ldots, x_{k}, x_{1} x_{2}, x_{2} x_{3}, \ldots, x_{k-1} x_{k}, x_{1} x_{2} x_{k-2} x_{k-1}, x_{1} x_{2} x_{k-1} x_{k} .
$$

Note that the connection set found in this lemma depends on the order as well as the choice of the elements in the generating set for $G$. We therefore use the following definition.

Definition 2.5. Let $G$ be an elementary abelian 2-group of rank $k \geq 6$. Given the generating tuple $\left(x_{1}, \ldots, x_{k}\right)$ for $G$, we refer to the connection set given in Eq. 2 as the Imrich generating set for $G$ with respect to $\left(x_{1}, \ldots, x_{k}\right)$.

In their work on the GRR problem, Nowitz and Watkins proved a lemma that is very useful in our context also. (Given a graph $\Gamma$ and a vertex $v$ of $\Gamma$, we denote by $\operatorname{Aut}(\Gamma)$ the automorphism group of $\Gamma$ and by $\operatorname{Aut}(\Gamma)_{v}$ the stabiliser of the vertex $v$ in $\operatorname{Aut}(\Gamma)$.)

Lemma 2.6 ((Nowitz and Watkins [10])). Let $G$ be a group, let $S$ be a subset of $G$, let $\Gamma:=\operatorname{Cay}(G, S)$ and let $X$ be a subset of $G$. If $\varphi$ fixes $X$ pointwise for every $\varphi \in \operatorname{Aut}(\Gamma)_{1}$, then $\varphi$ fixes $\langle X\rangle$ pointwise for every $\varphi \in \operatorname{Aut}(\Gamma)_{1}$.

Thus, if $\operatorname{Aut}(\Gamma)_{1}$ fixes every element of a generating set for $G$ or if the subgraph induced by $\Gamma$ on the neighbourhood $\Gamma(1)=S$ is asymmetric, then $\operatorname{Aut}(\Gamma)=G$. Hence $\Gamma$ is a DRR for $G$, and is therefore an ORR if the connection set satisfies $S \cap S^{-1}=\emptyset$. We will use this fact repeatedly when we cite the above lemma. Also, although Nowitz and Watkins did not make this explicit, the same proof applies if we replace both occurrences of the word "pointwise" in the statement of Lemma 2.6 with the word "setwise." We will also use this sometimes when we cite the above lemma.

We include in this section two more lemmas that we will need. Lemma 2.7 follows fairly easily from the work in [12, but we include a complete proof as the precise connection set (and therefore the fact that the induced subgraph on that connection set is weakly connected) is not easy to see from the statements in that paper.

Lemma 2.7. Let $A$ be an abelian 2-group of order $2^{k}$. Assume that $A$ is not elementary abelian and $A ¥ C_{4} \times C_{2}^{k-2}$. Then there is a generating set $S$ for $A$ with $|S| \geq 2$ such that the induced subgraph of $\Gamma:=\operatorname{Cay}(G, S)$ on $S$ is weakly connected with trivial automorphism group, so that $\Gamma$ is an ORR. 
Proof. From the structure of finite abelian groups, we may write $A=A_{1} \times \cdots \times A_{m}$, where $A_{i}:=\left\langle a_{i}\right\rangle$ is a non-trivial cyclic group for each $i \in\{1, \ldots, m\}$. Moreover, we may assume that $o\left(a_{i+1}\right)$ divides $o\left(a_{i}\right)$ for every $i \in\{1, \ldots, m-1\}$.

Since $A$ is not elementary abelian and $A \not C_{4} \times C_{2}^{k-2}$, we must have either $o\left(a_{1}\right)>4$, or $m \geq 2$ and $o\left(a_{1}\right)=o\left(a_{2}\right)=4$. In the first case, set $x_{1}:=a_{1}$, $x_{2}:=a_{1}^{-1} a_{2}$ and $x_{i}:=a_{1}^{(-1)^{i-1}} a_{i}$ for every $i \in\{3, \ldots, m\}$; in the second case, set $x_{1}:=a_{1}, x_{2}:=a_{2}, x_{2 i+1}:=a_{1} a_{2 i+1}$ and $x_{2 j}:=a_{2} a_{2 j}$ for every $2 i+1,2 j \in$ $\{3, \ldots, m\}$. In both cases, it is easy to verify that the set $X:=\left\{x_{1}, \ldots, x_{m}\right\}$ generates $A$ irredundantly. (In fact, $\left(x_{1}, \ldots, x_{m}\right)$ is a beautiful generating tuple for A.)

Consider $S:=X \cup Y$ where

$Y:= \begin{cases}\left\{x_{1}^{2}\right\} & \text { if } m=1, \\ \left\{x_{2} x_{1}^{-2}\right\} \cup\left\{x_{2} x_{1}^{-1}, x_{3} x_{2}^{-1}, \ldots, x_{m} x_{m-1}^{-1}\right\} & \text { if } m \geq 2 \text { and } o\left(a_{1}\right)>4 \\ \left\{x_{1} x_{2}\right\} \cup\left\{x_{2} x_{1}^{-1}, x_{3} x_{2}^{-1}, \ldots, x_{m} x_{m-1}^{-1}\right\} & \text { if } m \geq 2 \text { and } o\left(a_{1}\right)=o\left(a_{2}\right)=4 .\end{cases}$

Let $\Gamma:=\operatorname{Cay}(A, S)$ and observe that $S \cap S^{-1}=\emptyset$ so that $\Gamma$ is an oriented digraph.

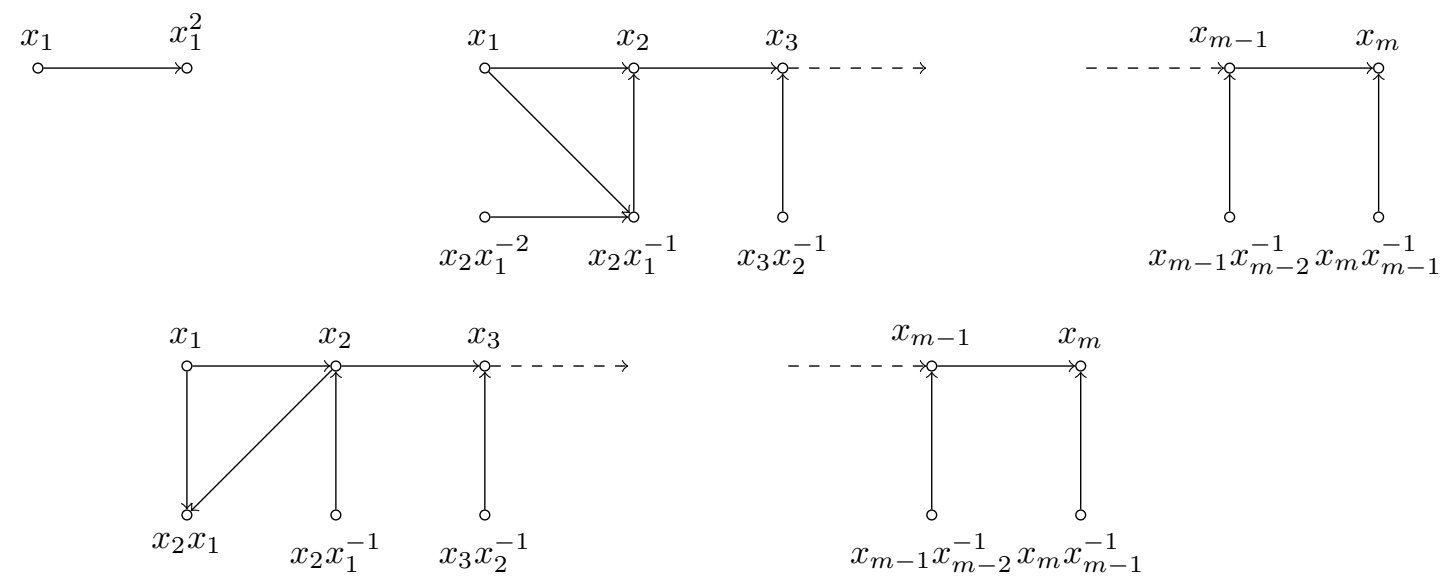

Figure 1. The oriented graph $\Delta$ in the proof of Lemma 2.7 top left when $m=1$; top right when $m \geq 2$ and $o\left(a_{1}\right)>4$; and bottom when $m \geq 2$ and $o\left(a_{1}\right)=o\left(a_{2}\right)=4$

Let $\Delta$ be the subgraph induced by $\Gamma$ on $S$. It is clear that $\left(x_{i-1}, x_{i}\right)$ and $\left(x_{i} x_{i-1}^{-1}, x_{i}\right)$ are arcs of $\Delta$ for every $i \in\{2, \ldots, m\}$. Moreover, when $m=1,\left(x_{1}, x_{1}^{2}\right)$ is an arc of $\Delta$; when $m \geq 2$ and $o\left(a_{1}\right)>4,\left(x_{1}, x_{2} x_{1}^{-1}\right)$ and $\left(x_{2} x_{1}^{-2}, x_{2} x_{1}^{-1}\right)$ are arcs of $\Delta$; while, when $m \geq 2$ and $o\left(a_{1}\right)=o\left(a_{2}\right)=4,\left(x_{1}, x_{1} x_{2}\right)$ and $\left(x_{2}, x_{1} x_{2}\right)$ are arcs of $\Delta$. (See Figure 1, This shows that $\Delta$ is weakly connected. It is not hard (but rather tedious because it requires some detailed computations) to show that these are the only arcs of $\Delta$. We do not give a complete proof of this easy fact, but we deal with one case to show the type of computations that are required. Suppose that there exists an arc $\left(x_{i} x_{i-1}^{-1}, x_{j} x_{j-1}^{-1}\right)$ between two distinct vertices in $Y$, for some $i, j \in\{2, \ldots, m\}$. Then $x_{j} x_{j-1}^{-1} x_{i-1} x_{i}^{-1} \in S$ and hence either $x_{j} x_{j-1}^{-1} x_{i-1} x_{i}^{-1}=x_{k}$ for some $k \in\{1, \ldots, m\}$, or $x_{j} x_{j-1}^{-1} x_{i-1} x_{i}^{-1}=x_{k} x_{k-1}^{-1}$ for some $k \in\{2, \ldots, m\}$, 
or $x_{j} x_{j-1}^{-1} x_{i-1} x_{i}^{-1} \in\left\{x_{1}^{2}, x_{1} x_{2}, x_{2} x_{1}^{-2}\right\}$. Each of these relations contradicts the irredundancy of the generating set $X$.

The structure of the arcs that we have described in $\Delta$ (see again Figure1) shows that $\Delta$ has trivial automorphism group. Lemma 2.6 then implies that $\Gamma$ is an ORR.

We conclude this section with a technical lemma. Although we will only apply this lemma with $|G: N| \leq 8$, we state it in greater generality as the proof is no different and the general result may be useful in future work on ORRs.

Lemma 2.8. Let $G$ be a 2-group and suppose that $G$ is not generalised dihedral. Let $N$ be a normal subgroup of $G$ with $G / N$ elementary abelian. Suppose that there exists $T \subseteq N$ with $|T| \geq 2$ such that $\operatorname{Cay}(N, T)$ is an ORR and such that the subgraph induced by $\operatorname{Cay}(N, T)$ on the neighbourhood $T$ of the vertex 1 is weakly connected. Let $\kappa:=d(G / N)$. Then there is a set of elements $\left\{a_{1}, \ldots, a_{\kappa}\right\} \subseteq G \backslash N$ such that:

- $G=\left\langle a_{1}, \ldots, a_{\kappa}, N\right\rangle ;$ and

- $o\left(a_{i}\right)>2$ for each $i \in\{1, \ldots, \kappa\}$.

Furthermore, if there exists such a set of elements with the additional property that, for every $i, j \in\{1, \ldots, \kappa\}$ with $i \neq j$, we have $a_{i}^{2}$ centralises $N$, and either $a_{i}^{2} \neq a_{j}^{2}$ or $a_{i} a_{j}$ does not centralise $N$, then $\operatorname{Cay}\left(G, T \cup\left\{a_{1}, \ldots, a_{\kappa}\right\}\right)$ is an ORR

Proof. Let $T \subseteq N$ satisfying the hypothesis of this lemma; that is: $|T| \geq 2$, $\operatorname{Cay}(N, T)$ is an ORR, and the subgraph induced by $\operatorname{Cay}(N, T)$ on the neighbourhood $T$ of the vertex 1 is weakly connected.

We begin by proving the first conclusion of this lemma. (The argument here is similar to the proof of [12, Lemma 2.6] and uses the ideas therein.) Among all $\kappa$-tuples $a_{1}, \ldots, a_{\kappa}$ of elements of $G$ such that $\left\{a_{1} N, \ldots, a_{\kappa} N\right\}$ is a generating set for $G / N$, choose one with as few involutions as possible. If no element in $\left\{a_{1}, \ldots, a_{\kappa}\right\}$ is an involution, then our first conclusion is proved. Hence we suppose that $\left\{a_{1}, \ldots, a_{\kappa}\right\}$ has at least one involution. Relabeling the index set $\{1, \ldots, \kappa\}$ if necessary, we may assume that $a_{1}$ is an involution.

Let $n \in N$. Now, $\left\{a_{1} n, a_{2}, \ldots, a_{\kappa}\right\}$ is still a generating set for $G$ modulo $N$ of cardinality $\kappa$. Since this generating set cannot contain fewer involutions than the original generating set, the element $a_{1} n$ must be an involution. Thus $1=\left(a_{1} n\right)^{2}=$ $a_{1} n a_{1} n=n^{a_{1}} n$, that is, $n^{a_{1}}=n^{-1}$. Since this argument does not depend upon $n \in N$, we obtain that $a_{1}$ acts by conjugation inverting each element of $N$. In particular, $N$ is abelian.

Let $j \in\{2, \ldots, \ell\}$ and let $n \in N$. Now, $\left\{a_{1} a_{j} n, a_{2}, a_{3}, \ldots, a_{\kappa}\right\}$ is still a generating set for $G$ modulo $N$ of cardinality $\kappa$. Since this generating set cannot contain fewer involutions than the original generating set, the element $a_{1} a_{j} n$ must be an involution. Thus

$$
1=\left(a_{1} a_{j} n\right)^{2}=a_{1} a_{j} n a_{1} a_{j} n=a_{1}^{2}\left(a_{j} n\right)^{a_{1}} a_{j} n=a_{j}^{a_{1}} n^{a_{1}} a_{j} n=a_{j}^{a_{1}} n^{-1} a_{j} n ;
$$

so $a_{j}^{a_{1}}=n^{-1} a_{j}^{-1} n$. By applying this equality with $n:=1$, we deduce that conjugation by $a_{1}$ inverts $a_{j}$. Therefore $a_{j}^{-1}=a_{j}^{a_{1}}=n^{-1} a_{j}^{-1} n$ for each $n \in N$, that is, $a_{j}$ commutes with $N$.

Let $i, j \in\{2, \ldots, \kappa\}$ with $i \neq j$. Arguing as above, $\left\{a_{1} a_{i} a_{j}, a_{2}, a_{3}, \ldots, a_{\ell}\right\}$ is still a generating set for $G$ modulo $N$ of cardinality $\kappa$. Since this generating set cannot 
contain fewer involutions than the original generating set, the element $a_{1} a_{i} a_{j}$ must be an involution. Thus

$$
1=\left(a_{1} a_{i} a_{j}\right)^{2}=a_{1} a_{i} a_{j} a_{1} a_{i} a_{j}=a_{1}^{2}\left(a_{i} a_{j}\right)^{a_{1}} a_{i} a_{j}=\left(a_{i}^{a_{1}} a_{j}^{a_{1}}\right) a_{i} a_{j}=\left(a_{i}^{-1} a_{j}^{-1}\right) a_{i} a_{j},
$$

so $a_{i} a_{j}=a_{j} a_{i}$; that is, $a_{i}$ and $a_{j}$ commute.

This shows that $M:=\left\langle N, a_{2}, \ldots, a_{\ell}\right\rangle$ is an abelian normal subgroup of $G$. Since $G=\left\langle M, a_{1}\right\rangle$ and $a_{1}$ has order 2 , we have $|G: M|=2$. Moreover, since the action of $a_{1}$ by conjugation inverts a generating set for $M$, we see that $G$ is a generalised dihedral group over $M$, contrary to our assumption. Our first conclusion is thus proven.

To complete the proof, we assume from now on that $\left\{a_{1}, \ldots, a_{\kappa}\right\}$ satisfies the additional hypothesis that, for every $i, j \in\{1, \ldots, \kappa\}$ with $i \neq j$, we have $a_{i}^{2}$ centralises $N$, and either: $a_{i}^{2} \neq a_{j}^{2}$, or $a_{i} a_{j}$ does not centralise $N$.

Consider the set $S:=T \cup\left\{a_{1}, \ldots, a_{\kappa}\right\}$. By construction, $S \cap S^{-1}=\emptyset$ and hence $\Gamma:=\operatorname{Cay}(G, S)$ is an oriented digraph. When $\kappa=0$, that is, $G=N, \Gamma$ is an ORR as part of our hypothesis. Thus, for the rest of the proof, we assume that $\kappa \geq 1$.

Let $\varphi$ be an automorphism of $\Gamma$ with $1^{\varphi}=1$. Now, $\varphi$ fixes the neighbourhood $\Gamma(1)=S$ of the vertex 1 , that is, $\varphi$ acts as a group of automorphisms of the subgraph $\Delta$ induced by $\Gamma$ on $S$. By construction, the vertices $a_{1}, \ldots, a_{\kappa}$ are isolated in $\Delta$. As $|T| \geq 2$ and $a_{1}, \ldots, a_{\kappa}$ are isolated in $\Delta, T$ is the (unique) connected component of $\Delta$ of largest possible order. Thus $\varphi$ fixes $T$ setwise. Therefore, by Lemma 2.6, $\varphi$ fixes $\langle T\rangle=N$ setwise. Thus $\varphi$ acts as an automorphism of the graph induced by $\Gamma$ on $N$; that is, on the Cayley digraph $\operatorname{Cay}(N, S \cap N)=\operatorname{Cay}(N, T)$. By hypothesis, Cay $(N, T)$ is an ORR and hence $\varphi$ fixes $N$ pointwise. Furthermore, this shows that, for every $n \in N$ and every vertex $v$ of $\Gamma$, we have

$$
(n v)^{\varphi}=n v^{\varphi} .
$$

Suppose that, for some $i \in\{1, \ldots, \kappa\}$, we have $a_{i}^{\varphi} \neq a_{i}$. Since $a_{i}^{\varphi} \in S$, there exists $j \in\{1, \ldots, \kappa\} \backslash\{i\}$ with $a_{i}^{\varphi}=a_{j}$. Now $\left(a_{i}, a_{i}^{2}\right)$ is an arc of $\Gamma$ and hence $\left(a_{i}^{\varphi},\left(a_{i}^{2}\right)^{\varphi}\right)$ is also an arc of $\Gamma$. Since $G / N$ is elementary abelian, we have $a_{i}^{2} \in N$ and, since $\varphi$ fixes $N$ pointwise, we have $\left(a_{i}^{2}\right)^{\varphi}=a_{i}^{2}$. Therefore, $a_{i}^{2}\left(a_{i}^{\varphi}\right)^{-1}=a_{i}^{2} a_{j}^{-1} \in$ $S \backslash N=\left\{a_{1}, \ldots, a_{\kappa}\right\}$. Since $d(G / N)=\kappa$, this forces $a_{i}^{2} a_{j}^{-1}=a_{j}$, so $a_{i}^{2}=a_{j}^{2}$.

By Eq. (3), $\left(n a_{i}\right)^{\varphi}=n a_{j}$ for every $n \in N$. Fix $n \in N$, and observe that $a_{i} n a_{i} \in N$ because $G / N$ is elementary abelian and that $\left(a_{i} n a_{i}\right)^{\varphi}=a_{i} n a_{i}$ because $\varphi$ fixes $N$ pointwise. Since $\left(n a_{i}, a_{i} n a_{i}\right)$ is an arc of $\Gamma,\left(\left(n a_{i}\right)^{\varphi},\left(a_{i} n a_{i}\right)^{\varphi}\right)=\left(n a_{j}, a_{i} n a_{i}\right)$ is also an arc of $\Gamma$; that is,

$$
a_{i} n a_{i}\left(n a_{j}\right)^{-1}=a_{i}^{2}\left(a_{i}^{-1} n a_{i}\right)\left(a_{j}^{-1} n^{-1} a_{j}\right) a_{j}^{-1}=a_{i}^{2} n^{a_{i}}\left(n^{-1}\right)^{a_{j}} a_{j}^{-1} \in S .
$$

As $a_{i}^{2} n^{a_{i}}\left(n^{-1}\right)^{a_{j}} a_{j}^{-1}$ lies in the coset $N a_{j}^{-1}$ and $G / N$ is elementary abelian, we deduce $a_{i}^{2} n^{a_{i}}\left(n^{-1}\right)^{a_{j}} a_{j}^{-1}=a_{j}$; that is, $n^{a_{i}}=n^{a_{j}}$. Thus $n^{a_{i} a_{j}}=\left(n^{a_{i}}\right)^{a_{j}}=n^{a_{j}^{2}}=n$ because $a_{j}^{2}$ centralises $N$ by hypothesis. As this argument does not depend upon $n \in N, a_{i} a_{j}$ centralises $N$, contradicting our hypothesis.

Since $i$ was arbitrary and our only assumption was that $\left(a_{i}\right)^{\varphi} \neq a_{i}$, we conclude that $\varphi$ fixes $S$ pointwise, so by Lemma 2.6 we have $\varphi=1$ and $\Gamma$ is an ORR. 


\section{Constructing ORRs in Groups that have LOW-INDEX ElEMEntary ABELIAN SUBGROUPS}

In this section, we prove some results that will be useful for constructing ORRs on many groups that have elementary abelian subgroups of low index. These results make intense use of Imrich generating sets for GRRs on elementary abelian groups of rank at least 6 , so they require the elementary abelian subgroup to be of sufficiently high rank.

Given a Cayley digraph $\operatorname{Cay}(G, S)$ and two vertices $x$ and $y$, we say that $x$ and $y$ are adjacent via $s$ if $y x^{-1}=s \in S$. Similarly, we say that $x$ and $y$ are adjacent via $\boldsymbol{X}$ if $y x^{-1} \in X \subset S$.

Lemma 3.1. Let $B$ be an elementary abelian 2-group of rank $k \geq 6$, and let $T$ be the Imrich generating set for $B$ (with respect to some generating tuple). Let $G$ be a group with $B \leq G$, let $B x$ be a coset of $B$ in $G$ and let $S$ be any subset of $G$ with $S \cap B x=T x \cup\{x\}$. Then in $\operatorname{Cay}(G, S), x$ is the only vertex of $S \cap B x$ that has at least three mutual inneighbours via elements of $S \cap B x$ with every other vertex of $S \cap B x$.

Proof. Let the generating tuple be $\left(z_{1}, \ldots, z_{k}\right)$. We begin by showing that $x$ has at least three common inneighbours via elements of $S \cap B x$ with every other vertex of $S \cap B x$. Let $t x$ be an arbitrary vertex of $(S \cap B x) \backslash\{x\}$, so $t \in T$. Observe that $y$ is a mutual inneighbour via elements of $S \cap B x$ of $x$ and $t x$ if and only if there exist $t_{1}, t_{2} \in T \cup\{1\}$ such that $t_{1} x y=x$ and $t_{2} x y=t x$. Equivalently, $y=t_{1}^{x}$ and $t_{1} t_{2}=t$. This is clearly satisfied with $y=t_{1}=1$ and $t_{2}=t$, so that 1 is a mutual inneighbour. It is also always satisfied by taking $y=t^{x}, t_{1}=t$, and $t_{2}=1$, so that $t^{x}$ is a mutual inneighbour. The third mutual inneighbour depends on $t$.

If $t=z_{i}$ for some $i \in\{1, \ldots, k-1\}$, then taking $t_{1}:=z_{i} z_{i+1}, t_{2}:=z_{i+1}$, and $y:=t_{1}^{x}$ gives $y$ as a third mutual inneighbour. If $t=z_{k}$, then taking $t_{1}:=z_{k-1} z_{k}$, $t_{2}:=z_{k-1}$, and $y:=t_{1}^{x}$ gives $y$ as a third mutual inneighbour. If $t=z_{i} z_{i+1}$ for some $i \in\{1, \ldots k-1\}$, then taking $t_{1}:=z_{i}, t_{2}:=z_{i+1}$, and $y:=t_{1}^{x}$ gives $y$ as a third mutual inneighbour. Finally, if $t=z_{1} z_{2} z_{\ell-1} z_{\ell}$ for $\ell \in\{k-1, k\}$, then taking $t_{1}:=z_{1} z_{2}, t_{2}:=z_{\ell-1} z_{\ell}$, and $y:=t_{1}^{x}$ gives $y$ as a third mutual inneighbour.

Now we show that for any vertex $t x$ of $T x$, there is some vertex $t^{\prime} x$ of $T x \backslash\{t x\}$ such that $t x$ and $t^{\prime} x$ have fewer than three common inneighbours via elements of $S \cap B x$. Observe that $y$ is a mutual inneighbour via elements of $S \cap B x$ of $t x$ and $t^{\prime} x$ if and only if there exist $t_{1}, t_{2} \in T \cup\{1\}$ such that $t_{1} x y=t x$ and $t_{2} x y=t^{\prime} x$. Equivalently, $y=\left(t_{1} t\right)^{x}$ and $t_{1} t_{2}=t t^{\prime}$. This is clearly satisfied with $y=1, t_{1}=t$, and $t_{2}=t^{\prime}$. It is also satisfied with $y=\left(t t^{\prime}\right)^{x}, t_{1}=t^{\prime}$, and $t_{2}=t$. Thus 1 and $\left(t t^{\prime}\right)^{x}$ are mutual inneighbours of $t x$ and $t^{\prime} x$.

If $t=z_{i}$ for some $i \in\{1, \ldots, k\}$, then let $t^{\prime}:=z_{j}$ for some $j \notin\{i-2, i-1, i, i+$ $1, i+2\}$ (such a $j$ exists since $k \geq 6$ ). There is no way to write $z_{i} z_{j}$ as a product of two elements $t_{1}, t_{2} \in T \cup\{1\}$ except when $\left\{t_{1}, t_{2}\right\}=\left\{z_{i}, z_{j}\right\}$. Thus there is no third mutual inneighbour of $z_{i} x$ and $z_{j} x$ via elements of $S \cap B x$. Similarly, if $t=z_{i} z_{i+1}$, then let $t^{\prime}$ be either $z_{i+2} z_{i+3}$ when $i \leq k-3$, or $z_{i-2} z_{i-1}$ otherwise. Again, if $t x$ and $t^{\prime} x$ had a third mutual inneighbour, then there would be some other way of writing some $z_{j} z_{j+1} z_{j+2} z_{j+3}$ (where $j \in\{i, i-2\}$ ) as a product of two elements of $T \cup\{1\}$, but this is not possible. Finally, if $t=z_{1} z_{2} z_{\ell-1} z_{\ell}$ where $\ell \in\{k-1, k\}$, then let $t^{\prime}:=z_{3}$. There is no other way of writing $z_{1} z_{2} z_{3} z_{\ell-1} z_{\ell}$ as a product of two elements of $T \cup\{1\}$. This completes the proof. 
Unfortunately, to make the above lemma directly useful in building ORRs, we would need to know that every automorphism of $\operatorname{Cay}(G, S)$ that fixes 1 also fixes $S \cap B x=T x \cup\{x\}$ setwise. This is challenging to prove in general. It turns out to be much easier to prove that if $S \cap B x=(B x \backslash T x) \backslash\{x\}$, then every automorphism of $\operatorname{Cay}(G, S)$ that fixes 1 also fixes $S \cap B x$. We therefore prove the following result about the case where $S \cap B x=(B x \backslash T x) \backslash\{x\}$, which is a corollary to the above lemma.

Corollary 3.2. Let $B$ be an elementary abelian 2-group of rank $k \geq 6$, and let $T$ be the Imrich generating set for $B$ (with respect to some generating tuple). Let $G$ be a group with $B \leq G$, let $B x$ be a coset of $B$ in $G$ and let $S$ be any subset of $G$ with $S \cap B x=(B x \backslash T x) \backslash\{x\}$. If $\operatorname{Aut}(\operatorname{Cay}(G, S))_{1}$ fixes $S \cap B x$ setwise, then $\operatorname{Aut}(\operatorname{Cay}(G, S))_{1}$ fixes $x$.

Proof. Assume that, for every $\varphi \in \operatorname{Aut}(\operatorname{Cay}(G, S))_{1},(S \cap B x)^{\varphi}=S \cap B x$. Observe first that $B^{x}$ is uniquely determined setwise as the set of vertices that have an outneighbour in $S \cap B x$ via some element of $S \cap B x$. Furthermore, $X:=T x \cup\{x\}$ is uniquely determined as the set of vertices that are not in $S$, but are outneighbours of some vertex in $B^{x}$ via some element of $S \cap B x$. Now Lemma 3.1 (applied with the set $S$ replaced by $G \backslash S$ ) tells us that $x$ is the only vertex in $X$ having the property that, for every other vertex $y \in X$, there are at least three vertices of $B^{x}$ that are not inneighbours of either $y$ or $x$. Thus $x^{\varphi}=x$, for every $\varphi \in \operatorname{Aut}(\operatorname{Cay}(G, S))_{1}$.

Finally, we can describe a situation that will arise frequently in which $S \cap B x=$ $(B x \backslash T x) \backslash\{x\}$ is fixed setwise by every automorphism $\varphi$ of $\operatorname{Cay}(G, S)$ that fixes 1. We show that in this situation, $B$ and $x$ are fixed pointwise by $\varphi$, which is a significant step toward proving that $\operatorname{Cay}(G, S)$ is an ORR.

Proposition 3.3. Let $G$ be a group, and suppose that $B<G$ where $B$ is an elementary abelian group of rank $k \geq 6$, and $x \in G$ centralises $B$. Let $T$ be the Imrich generating set for $B$ with respect to some generating tuple, and let $S$ be a generating set for $G$ such that $S=[(B x \backslash T x) \backslash\{x\}] \cup X$, where $X \subseteq G \backslash\langle B, x\rangle$ with $|X| \leq 17$. Then $\operatorname{Aut}(\operatorname{Cay}(G, S))_{1}$ fixes $B$ and $x$ pointwise.

Proof. We claim that a vertex of $\operatorname{Cay}(G, S)$ is an outneighbour of at least $2^{k}-4 k-4$ vertices of $S$ if and only if it is an element of $B x^{2}$.

Let $b x^{2}$ be an arbitrary element of $B x^{2}$. Then, for any $c \in(B \backslash T) \backslash\{1\}$, we have $b c \in B$, so $(b c x)(c x)=b x^{2}$ is an outneighbour of $c x$ unless $b c \in T \cup\{1\}$; that is, unless $c \in b(T \cup\{1\})$. Since there are $2 k+2$ elements in $b(T \cup 1)$, there are at most $2 k+2$ vertices in $(B x \backslash T x) \backslash\{x\}$ that are not inneighbours of $b x^{2}$. This means that there are at least $2^{k}-4 k-4$ vertices in $S$ that are inneighbours of $b x^{2}$.

On the other hand, if we take a vertex $v$ that is not in $B x^{2}$ and it is an outneighbour of at least $2^{k}-4 k-4$ vertices of $S$, then since $2^{k}-4 k-4>17, v$ must be an outneighbour of some vertex $b x \in B x \cap S$. As we observed above, outneighbours of vertices of $B x$ via elements of $S \cap B x$ are in $B x^{2}$, so $v \notin B x^{2}$ implies that $v$ is an outneighbour of at most 17 vertices that are in $B x$. Therefore, $v$ is an outneighbour of at most 34 vertices of $S$ (at most 17 in $B x$ and at most 17 in $X$ ). Since $2^{k}-4 k-4>34$, this completes the proof of our claim.

Let $\varphi \in \operatorname{Aut}(\operatorname{Cay}(G, S))_{1}$. The previous paragraph shows that $\left(B x^{2}\right)^{\varphi}=B x^{2}$. Since $B x$ is the set of vertices of $\operatorname{Cay}(G, S)$ such that all but at most 17 of their outneighbours lie in $B x^{2}$, we also have $(B x)^{\varphi}=B x$. Thus $(S \cap B x)^{\varphi}=S \cap B x=$ $(B x \backslash T x) \backslash\{x\}$. 
Applying Corollary 3.2 to our graph, we see that $x^{\varphi}=x$, and so $(T x)^{\varphi}=T x$. Since $T$ is the connection set for a GRR on $B$ and $x$ is fixed, this implies that $B x$ is fixed pointwise, which means that (using Lemma 2.6) $B$ is fixed pointwise.

We will be using this proposition for most groups $G$ arising from Theorem 2.3 that contain an elementary abelian subgroup of low index. As long as $G$ is sufficiently large, the elementary abelian subgroup will have high enough rank to allow us to apply this result. For small orders of $G$, we will make use of computations in magma to complete the proof.

\section{The groups in Theorem 2.3 (ii)}

In this section we deal with the groups arising from Theorem 2.3 (ii). First, we give a result that finds an ORR for all but three specific families of groups.

Proposition 4.1. Let $G$ be a 2-group with an abelian subgroup $A$, with a normal subgroup $N$ and with two elements $g \in G \backslash N$ and $n \in N \backslash A$ such that

$A<N<G,|G: N|=|N: A|=2, g^{2}=1, n^{g}=n^{-1}$, and $a^{g}=a^{-1}$, for each $a \in A$.

Then one of the following holds:

(a) G has an ORR;

(b) $\ell$ and $\kappa$ are non-negative integers, $V$ is an elementary abelian 2-group of rank $2 \ell+\kappa$ with generating set

$$
\left\{v_{1}, w_{1}, \ldots, v_{\ell}, w_{\ell}, e_{1}, \ldots, e_{\kappa}\right\}
$$

and $G=\langle V, x\rangle$, where $v_{i}^{x}=w_{i}, w_{i}^{x}=v_{i}$ for $i \in\{1, \ldots, \ell\}$ and $e_{i}^{x}=e_{i}$ for $i \in\{1, \ldots, \kappa\} ;$ and

(i) $x^{2}=1$; or

(ii) $\kappa \geq 1$ and $x^{2}=e_{1}$;

(c) $G$ contains a maximal subgroup $D$ isomorphic to $D_{4} \times C_{2}^{\ell}$ for some $\ell \in \mathbb{N}$ $\left(D_{4}\right.$ is the dihedral group of order 8); or

(d) $G$ is generalised dihedral.

Proof. We assume that conclusion (d) does not hold, that is, $G$ is not generalised dihedral.

Suppose that $A$ has exponent 2. Then $V:=\langle A, g\rangle$ is a maximal subgroup of $G$ and is elementary abelian. Now, $V$ is a $\mathbb{Z}\langle n\rangle$-module. Therefore, from the structure theorem of finitely generated $\mathbb{Z}\langle n\rangle$-modules, we may write $V=A_{1} \times A_{2} \times \cdots \times A_{\ell+\kappa}$, where $A_{i}:=\left\langle v_{i}\right\rangle$ is a non-identity non-trivial cyclic $\mathbb{Z}\langle n\rangle$-module for each $i \in$ $\{1, \ldots, \ell\}$, and $A_{\ell+i}:=\left\langle e_{i}\right\rangle$ is a non-identity trivial (and hence cyclic) $\mathbb{Z}\langle n\rangle$-module for each $i \in\{1, \ldots, \kappa\}$. For each $i \in\{1, \ldots, \ell\}$, write $w_{i}:=v_{i}^{n}$. As $n^{2} \in A$ centralises $V, v_{1}, w_{1}, v_{2}, w_{2}, \ldots, v_{\ell}, w_{\ell}, e_{1}, \ldots, e_{\kappa}$ is a basis of $V$ (as a vector space over the field of cardinality 2$)$ and $|V|=2^{2 \ell+\kappa}$.

Now the action of $n$ on $V$ is encoded in the pair $(\ell, \kappa)$. Thus, to determine the isomorphism class of $G$ it suffices to determine $n^{2}$. Write $n^{2}=v_{1}^{\varepsilon_{1}} w_{1}^{\varepsilon_{1}^{\prime}} \cdots v_{\ell}^{\varepsilon_{\ell}} w_{\ell}^{\varepsilon_{\ell}^{\prime}} e_{1}^{\eta_{1}} \cdots e_{\kappa}^{\eta_{\kappa}}$, for some $\varepsilon_{1}, \varepsilon_{1}^{\prime}, \ldots, \varepsilon_{\ell}, \varepsilon_{\ell}^{\prime}, \eta_{1}, \ldots, \eta_{\kappa} \in\{0,1\}$. Since $n$ centralizes $n^{2}$ and $v_{i}^{n}=w_{i}$, $w_{i}^{n}=v_{i}$, we deduce that $\varepsilon_{i}=\varepsilon_{i}^{\prime}$ for every $i \in\{1, \ldots, \ell\}$. Let $I:=\{i \in\{1, \ldots, \ell\} \mid$ $\left.\varepsilon_{i}=1\right\}$ and write $n^{\prime}:=n \prod_{i \in I} v_{i}$. Now, $n$ and $n^{\prime}$ induce the same action by conjugation on $V$ and $\left(n^{\prime}\right)^{2}=e_{1}^{\eta_{1}} \cdots e_{\kappa}^{\eta_{\kappa}}$. In particular, replacing $n$ by $n^{\prime}$ if necessary, we may assume that $\varepsilon_{i}=0$ for each $i \in\{1, \ldots, \ell\}$. 
If $\eta_{1}=\cdots=\eta_{\kappa}=0$, then $n^{2}=1$ and $G$ splits over $V$. Taking $x:=n$, this is conclusion (b)(i).

If $\eta_{i} \neq 0$ for some $i \in\{1, \ldots, \kappa\}$, then $n^{2} \neq 1$ and $e_{1}, \ldots, e_{i-1}, n^{2}, e_{i+1}, \ldots, e_{\kappa}$ are linearly independent and span $\left\langle e_{1}, \ldots, e_{\kappa}\right\rangle$. Therefore, up to a change of basis in $\left\langle e_{1}, \ldots, e_{\kappa}\right\rangle$, we may assume that $n^{2}=e_{1}$. This is conclusion (b)(ii).

Suppose that $A \cong C_{4} \times C_{2}^{\ell}$, for some $\ell \in \mathbb{N}$. Now $D:=\langle A, g\rangle$ is generalised dihedral isomorphic to $D_{4} \times C_{2}^{\ell}$. This is conclusion (c).

For the rest of the proof, we may assume that $A$ is neither elementary abelian nor isomorphic to $A \cong C_{4} \times C_{2}^{\ell}$, for some $\ell \in \mathbb{N}$. By Lemma 2.7, there exists $T \subseteq A$ with $|T| \geq 2$ such that $\operatorname{Cay}(A, T)$ is an ORR and such that the induced subgraph of $\operatorname{Cay}(A, T)$ on $T$ is weakly connected. As $G / A$ is elementary abelian of order 4 and $G$ is not generalised dihedral, by Lemma 2.8 applied to the normal subgroup $A$, there exist $a_{1}, a_{2} \in G \backslash N$ with $G=\left\langle a_{1}, a_{2}, N\right\rangle, o\left(a_{1}\right)>2$ and $o\left(a_{2}\right)>2$. Since $G \backslash A=n A \cup g A \cup n g A$ and every element of $g A$ is an involution, the set $\left\{a_{1}, a_{2}\right\}$ consists of one element of $n A$ and one of $n g A$. Since $G / A$ is elementary abelian and $A$ is abelian, $a_{1}^{2}$ and $a_{2}^{2}$ are in $A$ and both centralise $A$. Now $a_{1} a_{2} \in g A$ and no element of $g A$ centralises $A$, because $A$ does not have exponent 2 . Thus, the second hypothesis of Lemma 2.8 holds, and we deduce that $G$ admits an ORR; that is, conclusion (a) holds.

We break the rest of this section into subsections, each dealing with one of the three families of groups described in this proposition that are not generalised dihedral, but have not yet been shown to admit ORRs.

4.1. The groups of Proposition 4.1)(b)(i). Let $G$ be a group that arises in Proposition 4.1(b)(i). Then we have $G=V \rtimes\langle x\rangle$, where $V=\left\langle v_{1}, w_{1}, \ldots, v_{\ell}, w_{\ell}, e_{1}, \ldots, e_{\kappa}\right\rangle$ is an elementary abelian 2-group, $x^{2}=1, e_{i}^{x}=e_{i}$ for every $i \in\{1, \ldots, \kappa\}$, and $v_{i}^{x}=w_{i}$ for every $i \in\{1, \ldots, \ell\}$.

We begin by observing that, if $\ell \leq 1$, then $G$ is a generalised dihedral group.

Lemma 4.2. Let $G$ be a group that arises in Proposition $4.1(b)(i)$. If $\ell \leq 1$, then $G$ is generalised dihedral.

Proof. If $\ell=0$, then $G$ is elementary abelian, which is a special form of generalised dihedral. If $\ell=1$, then $V^{\prime}=\left\langle v_{1} x, e_{1}, \ldots, e_{\kappa}\right\rangle$ is an abelian group having index 2 in $G$ (since $\left.\left(v_{1} x\right)^{2}=v_{1} w_{1}\right)$. Also, the action of $w_{1}$ by conjugation inverts every element of $V^{\prime}$. Thus, $G$ is a generalised dihedral group over $V^{\prime}$.

We now show that there is an ORR for any remaining sufficiently large group of this type.

Lemma 4.3. Let $G$ be a group that arises in Proposition $4.1(b)(i)$. If $\ell \geq 2$ and $2 \ell+\kappa \geq 8$, then $G$ has an ORR.

Proof. We will use two different generating sets, according to whether or not $\kappa \geq 2$, or $\kappa \in\{0,1\}$.

If $\kappa \geq 2$, then let

$$
V_{1}:=\left\langle v_{2}, \ldots, v_{\ell}, w_{2}, \ldots, w_{\ell}, e_{1}, \ldots, e_{\kappa}\right\rangle
$$

and let $T_{1}$ be the Imrich generating set for $V_{1}$ with respect to this generating tuple (this exists because the rank of $V_{1}$ is $2(\ell-1)+\kappa \geq 6$ ). If $\kappa \in\{0,1\}$, then $2 \ell+\kappa \geq 8$ 
implies $\ell \geq 4$. In this case, let

$$
V_{2}:=\left\langle v_{2}, v_{4} w_{4}, v_{3}, v_{2} w_{2}, v_{4}, v_{2} w_{3}, v_{5}, \ldots, v_{\ell}, e_{1}, \ldots, e_{\kappa}\right\rangle
$$

and let $T_{2}$ be the Imrich generating set for $V_{2}$ with respect to this generating tuple (this exists because the rank of $V_{2}$ is at least 6 since the first 6 elements are always in the tuple).

Let

$$
S:=\left[\left(V_{i} \backslash T_{i}\right) \backslash\{1\}\right] v_{1} x \cup\left\{v_{2} x\right\},
$$

where $i=1$ if $\kappa \geq 2$ and $i=2$ if $\kappa \in\{0,1\}$.

Observe that the product of any two elements of $S$ has the form $v v_{1} x v^{\prime} v_{1} x=$ $v v_{1}\left(v^{\prime}\right)^{x} w_{1} \in V_{1} v_{1} w_{1}$ (where $v, v^{\prime} \in V_{i}$ ), or $v v_{1} x v_{2} x=v v_{1} w_{2} \in V_{1} v_{1}$ (where $v \in V_{i}$ ), or $v_{2} x v v_{1} x=v_{2} v^{x} w_{1} \in V_{1} w_{1}$ (where $v \in V_{i}$ ), or $\left(v_{2} x\right)^{2}=v_{2} w_{2}$. Since none of these can be $1, S$ is the connection set for an oriented digraph.

Claim: $v_{2} x$ is the only vertex of $S$ for which all of its outneighbours have no other inneighbour from $S$.

Proof of the Claim: Observe that the outneighbours of $v_{2} x$ are all in $V_{1} v_{1} \cup V_{1}$, while the outneighbours of any vertex of $V_{i} v_{1} x$ are in $V_{1} v_{1} w_{1} \cup V_{1} w_{1}$. Thus every outneighbour of $v_{2} x$ has a unique inneighbour from $S$. It remains to prove the uniqueness.

Let $v v_{1} x \in S$ be arbitrary, so that $v \in V_{i}$ but $v \notin T_{i} \cup\{1\}$. If $i=1$, then let $f_{1}:=e_{1}$ and $f_{2}:=e_{2}$; if $i=2$, then let $f_{1}:=v_{2} w_{2}$ and $f_{2}:=v_{3} w_{3}$. We claim that $v \notin\left(T_{i} \cup\{1\}\right) f_{1} \cap\left(T_{i} \cup\{1\}\right) f_{2}$. Otherwise, $v=t f_{1}=t^{\prime} f_{2}$ for some $t, t^{\prime} \in T_{i} \cup\{1\}$ implies $t t^{\prime}=f_{1} f_{2}$. Due to the structure of $T_{2}$, this has no solutions if $i=2$. When $i=1$, the only solutions are $\left\{t, t^{\prime}\right\}=\left\{1, e_{1} e_{2}\right\}$ or $\left\{t, t^{\prime}\right\}=\left\{e_{1}, e_{2}\right\}$. Thus $v \in\left\langle e_{1}, e_{2}\right\rangle \subset T_{1} \cup\{1\}$, a contradiction that completes the proof of the claim.

Let $j \in\{1,2\}$ be such that $v \notin\left(T_{i} \cup\{1\}\right) f_{j}$. Then $v f_{j} \notin T_{i} \cup\{1\}$, so $v f_{j} v_{1} x \in S$ and $\left(v f_{j} v_{1} x\right)^{2}=v_{1} w_{1} v v^{x}$ is an outneighbour of $v f_{j} v_{1} x$. It is also an outneighbour of $v v_{1} x$ since $\left(v v_{1} x\right)^{2}=v_{1} w_{1} v v^{x}$.

Let $\varphi \in \operatorname{Aut}(\operatorname{Cay}(G, S))_{1}$. From the previous claim, $\varphi$ fixes $v_{2} x$. This implies that $V_{i} v_{1} x \cap S$ is fixed setwise by $\varphi$. Now we can apply Corollary 3.2 to see that $\varphi$ fixes $v_{1} x$. This also implies that $\left(T_{i} v_{1} x\right)^{\varphi}=T_{i} v_{1} x$, and our choice of $T_{i}$ as the generating set for a GRR on $V_{i}$ implies that $V_{i} v_{1} x$ is fixed pointwise by $\varphi$, and therefore so is $V_{i}$. Thus $\varphi$ fixes pointwise the generating set $\left\{v_{2} x, v_{1} x\right\} \cup V_{i}$ of $G$. By Lemma 2.6, Cay $(G, S)$ is an ORR.

This shows that every group that arises in Proposition 4.1(b)(i) with $\ell \geq 2$ that has order at least $2^{9}$ has an ORR.

4.2. The groups of Proposition 4.1(b)(ii). Let $G$ be a group that arises in Proposition 4.1(b)(ii). Then we have $G=\langle V, x\rangle$, where $V=\left\langle v_{1}, w_{1}, \ldots, v_{\ell}, w_{\ell}, e_{1}, \ldots, e_{\kappa}\right\rangle$ is an elementary abelian 2-group, $x^{2}=e_{1}, e_{i}^{x}=e_{i}$ for every $i \in\{1, \ldots, \kappa\}$, and $v_{i}^{x}=w_{i}$ for every $i \in\{1, \ldots, \ell\}$.

Lemma 4.4. Let $G$ be a group that arises in Proposition 4.1(b)(ii). If $2 \ell+\kappa \geq 7$, then $G$ has an ORR.

Proof. Let $\left(v_{1}, \ldots, v_{\ell}, w_{1}, \ldots, w_{\ell}, e_{2}, \ldots e_{\kappa}\right)$ be a generating tuple for

$$
V^{\prime}:=\left\langle v_{1}, \ldots, v_{\ell}, w_{1}, \ldots, w_{\ell}, e_{2}, \ldots e_{\kappa}\right\rangle<V
$$


and let $T$ be the Imrich generating set for $V^{\prime}$ with respect to this generating tuple (this exists because $V^{\prime}$ has rank $2 \ell+\kappa-1 \geq 6$ ). Let

$$
S:=T x \cup\{x\} .
$$

As the product of any two elements of $S$ is not 1 (since it will be in $V^{\prime} e_{1}$ ), $\operatorname{Cay}(G, S)$ is an oriented Cayley digraph.

By Lemma 3.1, $x$ is the unique vertex of $S$ that has at least three mutual inneighbours with every other vertex of $S$. Since any automorphism $\varphi \in \operatorname{Aut}(\operatorname{Cay}(G, S))_{1}$ fixes $S$ setwise, we must have $x^{\varphi}=x$. Furthermore $(T x)^{\varphi}=T x$ and since $T$ is the generating set for a GRR on $V^{\prime}$, any automorphism that fixes $x$ and fixes $T x$ setwise must actually fix $\langle T\rangle x=V^{\prime} x$ pointwise. Since $\varphi$ fixes every point of $\left\{x, V^{\prime} x\right\}$ and $\left\langle x, V^{\prime} x\right\rangle=G$, by Lemma 2.6 we have $\varphi=1$ and this completes the proof.

This shows that every group that arises in Proposition 4.1(b)(ii) that has order at least $2^{8}$ has an ORR.

\subsection{The groups of Proposition 4.1(c).}

Notation 4.5. Let $G$ be a group that arises in Proposition 4.1(c). Then $G$ has a maximal subgroup $D$ isomorphic to $D_{4} \times C_{2}^{\ell}$ for some $\ell \in \mathbb{N}$. Studying the initial description of the groups in Proposition 4.1, we see that we may assume that $G=\langle A, g, n\rangle$, where $A \cong C_{4} \times C_{2}^{k}$ is generated by $a_{1}, \ldots, a_{k+1}$ with $o\left(a_{1}\right)=4$ and $o\left(a_{i}\right)=2$ for $i \in\{2, \ldots, k+1\}$, and $g$ acts by conjugation on $A$ inverting every $a_{i}, g^{2}=1, n^{g}=n^{-1}, n$ normalises $A$ and $n^{2} \in A$. Furthermore, if $n$ centralises $A$, then $N:=\langle A, n\rangle$ is abelian and $G=\langle N, g\rangle$ is generalised dihedral. Thus, if we assume that $G$ is not generalised dihedral, $n$ does not centralise $A$.

Lemma 4.6. Let $G$ be as in Notation 4.5. If $G$ is not generalised dihedral, then there exists $a \in A$ such that $o(a)>2$ and $a$ is not centralised by $n$.

Proof. Assume that $G$ is not generalised dihedral. Therefore $n$ must not centralise $A$. Also, there is some $a_{1} \in A$ with $o\left(a_{1}\right)>2$. If $a_{1}$ is not centralised by $n$ then taking $a:=a_{1}$ we are done, so we may assume that $n$ centralises every element of $A$ of order greater than 2. Since $n$ does not centralise $A$, there is some $b \in A$ such that $b$ is not centralised by $n$. Thus $o(b)=2$. Let $c:=b^{n}$, so $c \neq b$ has order 2 . Now, $a_{1} b$ has order $o\left(a_{1}\right)>2$, and $\left(a_{1} b\right)^{n}=a_{1} b^{n}=a_{1} c \neq a_{1} b$, so taking $a:=a_{1} b$ satisfies our conclusion.

Lemma 4.7. Let $G$ be as in Notation 4.5. If o $n)=2$ and $G$ is not generalised dihedral, then there is some element $a \in A$ such that $o(a)>2$ and $a$ is not inverted by $n$.

Proof. Assume $o(n)=2$. Let $a_{1}$ be an element of $A$ with $o\left(a_{1}\right)>2$. If $a_{1}$ is not inverted by $n$ then taking $a:=a_{1}$ we are done, so we may assume that $n$ inverts every element of $A$ of order greater than 2. Consider $a_{1} b$ for any $b$ of order 2 in $A$. As $o\left(a_{1} b\right)=o\left(a_{1}\right)>2$, we have $a_{1}^{-1} b=\left(a_{1} b\right)^{-1}=\left(a_{1} b\right)^{n}=a_{1}^{n} b^{n}=a_{1}^{-1} b^{n}$, and $b^{n}=b$. Thus $n$ centralises every element of order 2 , and this implies that every element in $A$ is inverted by $n$. Hence $n g$ centralises $A$ and $\langle A, n g\rangle$ is abelian. Since the action of $g$ by conjugation inverts $n g$ (because $(n g)^{g}=n^{g} g=n^{-1} g=n g$ ) and each element of $A$, we deduce that $G$ is a generalised dihedral group over $\langle A, n g\rangle$, a contradiction. 
We now have the key points for dealing with these groups. Our final lemma in this section shows that all sufficiently large groups that arise in Proposition 4.1(c) are either generalised dihedral or have ORRs.

Lemma 4.8. Let $G$ be as in Notation 4.5, with $k \geq 6$. Then either $G$ is generalised dihedral, or $G$ admits an ORR.

Proof. Suppose that $G$ is not generalised dihedral; let $B:=\left\langle a_{2}, \ldots, a_{k+1}\right\rangle$, and let $T$ be the Imrich generating set for $B$ with respect to some generating tuple. By Lemma 4.6. there exists $a \in A$ with $o(a)>2$ and $a^{n} \neq a$. Let

$$
S:= \begin{cases}{[(B a \backslash T a) \backslash\{a\}] \cup\left\{n^{-1}, a n^{-1} g, a n\right\}} & \text { when } a^{n} \neq n^{-2} a^{-1} \text { and } o(n) \neq 2 ; \\ {[(B a \backslash T a) \backslash\{a\}] \cup\left\{a n^{-1} g, a n\right\}} & \text { when } a^{n} \neq a^{-1} \text { and } o(n)=2 ; \\ {[(B a \backslash T a) \backslash\{a\}] \cup\left\{a n^{-1} g, n\right\}} & \text { when }\left(a^{\prime}\right)^{n}=n^{-2}\left(a^{\prime}\right)^{-1} \text { for every } a^{\prime} \in A \text { with } o\left(a^{\prime}\right)=4 .\end{cases}
$$

Observe that the three cases above do not cover all possibilies: in the remaining case every element $a^{\prime}$ of order 4 in $A$ is either centralised by $n$, or has $\left(a^{\prime}\right)^{n}=n^{-2}\left(a^{\prime}\right)^{-1}$, and they are not all in the second category. In the next paragraph, we study this latter possibility.

So there is $a \in A$ as in Lemma 4.6, and $a^{n}=n^{-2} a^{-1}$. Notice that $a \neq a^{n}=$ $n^{-2} a^{-1}$ implies that $n^{2} \neq a^{2}$ in this case.

ClaIM: There exist three elements $b_{1}, b_{2}, b_{3} \in B$ such that $a b_{1}, a b_{1} b_{2}, a b_{1} b_{3}$ are distinct and centralised by $n$.

Proof of claim: Since not all elements of order 4 of $A$ are in the second category, there exists an element of order 4 centralised by $n$. As $A=\langle a\rangle \times B$, we may assume that this element is of the form $a b_{1}$, for some $b_{1} \in B \backslash\{1\}$. If $\left|\mathbf{C}_{B}(n)\right|>2$, then we may choose two distinct elements $b_{2}, b_{3} \in \mathbf{C}_{B}(n) \backslash\{1\}$, and now $n$ centralises the three distinct elements $a b_{1}, a b_{1} b_{2}, a b_{1} b_{3}$. Assume $\left|\mathbf{C}_{B}(n)\right| \leq 2$.

Now, $B_{0}:=\left\langle a^{2}, B\right\rangle$ is characteristic in $A$ because it consists of all elements of order 2 of $A$, and hence $B_{0}$ is $n$-invariant. Clearly, $\left|\mathbf{C}_{B_{0}}(n)\right| \leq 2\left|\mathbf{C}_{B}(n)\right| \leq 4$. As $n^{2} \in A$ centralises $A$, the mapping $f: B_{0} \rightarrow \mathbf{C}_{B_{0}}(n)$ defined by $x \mapsto x x^{n}$ is a homomorphism with kernel $\mathbf{C}_{B_{0}}(n)$. Therefore, from the first homomorphism theorem, $\left|B_{0} / \mathbf{C}_{B_{0}}(n)\right| \leq\left|\mathbf{C}_{B_{0}}(n)\right|$ and $\left|B_{0}\right| \leq\left|\mathbf{C}_{B_{0}}(n)\right|^{2}=16$. However, $\left|B_{0}\right|=$ $2^{k+1} \geq 2^{6+1}$, a contradiction

Let $b_{1}, b_{2}, b_{3} \in B$ be as in the previous claim and let

$$
S:=[(B a \backslash T a) \backslash\{a\}] \cup\left\{a n^{-1} g, a b_{1} n, a b_{1} b_{2} n, a b_{1} b_{3} n\right\} .
$$

We claim that, in all four cases, $\Gamma:=\operatorname{Cay}(G, S)$ is an ORR for $G$. First we show that $S$ is asymmetric and all of its elements have order greater than 2. Certainly in every case the elements of $S \cap A$ have order 4 and do not contain any inverse-closed pair. Any products that are not in $A$ will clearly not be 1 . The remaining pairwise products are $n^{-1}$ an $=a^{n} \neq 1 ; a n n^{-1}=a \neq 1 ; n^{-2}$ or $n^{2}$ (notice that, if $o(n)=2$, then Lemma 4.7 implies that $n \notin S) ;\left(a n^{-1} g\right)^{2}=a\left(a^{n}\right)^{-1} \neq 1$ by our choice of $a$; $(a n)^{2}=a a^{n} n^{2} \neq 1$ by our choice of $a$ in the cases where an $\in S$; and for every $b_{i}, b_{j} \in\left\{1, b_{1}, b_{2}, b_{3}\right\}$, we have $a b_{1} b_{i} n a b_{1} b_{j} n=a^{2} b_{i} b_{j} n^{2} \neq 1$ since $n^{2} \neq a^{2} b_{i} b_{j}$. Thus $\Gamma$ is an oriented digraph.

Let $\varphi \in \operatorname{Aut}(\Gamma)_{1}$. By Proposition 3.3, $a^{\varphi}=a$ and $\varphi$ fixes every element of $B$ pointwise. Therefore $\varphi$ fixes every element of $\langle a, B\rangle=A$ pointwise.

Now if $S$ has the first of the four possible forms, then $a n^{-1} g$ is the only vertex of $\left\{a n^{-1} g, a n, n^{-1}\right\}$ that has only one outneighbour (via these three elements) in 
$A$, so $\left(a n^{-1} g\right)^{\varphi}=a n^{-1} g$. The outneighbours of $n^{-1}$ via $\left\{n^{-1}, a n\right\}$ are $n^{-2}$ and $a$, while the outneighbours of $a n$ are $a^{n}$ and $a a^{n} n^{2}$. We know that $a^{n} \neq a$, and $a^{n}=n^{-2}$ implies $n^{-2}=a$, but then $a^{n}=a$, a contradiction. Thus an is unique in having $a^{n}$ as an outneighbour, so $(a n)^{\varphi}=a n$. This completes the proof since $\varphi$ fixes every element of the generating set $\left\{a n^{-1} g, a n\right\} \cup A$ of $G$.

If $S$ has the second of the four possible forms, then $n^{2}=1$ and the outneighbours of $a n^{-1} g$ via $a n^{-1} g$ and $a n$ are $a\left(a^{n}\right)^{-1}$ and $a n a n^{-1} g=a a^{n} g$, while the outneighbours of $a n$ are $a a^{n}$ and $a n^{-1}$ gan $=a\left(a^{n}\right)^{-1} g$. Thus $a n$ is the only one of these vertices that has $a\left(a^{n}\right) \in A$ as an outneighbour, so $(a n)^{\varphi}=a n$ and $\left(a n^{-1} g\right)^{\varphi}=a n^{-1} g$. This completes the proof since $\varphi$ fixes every element of the generating set $\left\{a n, a n^{-1} g\right\} \cup A$ of $G$.

If $S$ has the third of the four possible forms, then $\left(a^{\prime}\right)^{n}=n^{-2}\left(a^{\prime}\right)^{-1}$ for every $a^{\prime}$ of order 4 in $A$. The pairwise products of $\left\{a n^{-1} g, n\right\}$ are $a\left(a^{n}\right)^{-1}=a\left(n^{-2} a^{-1}\right)^{-1}=$ $n^{2} a^{2}, a n^{-2} g, n^{2}$, and $a^{n} g$. Since $a^{2} \neq 1, a n^{-1} g$ and $n$ do not have the same outneighbours, so $n^{\varphi}=n$ and $\left(a n^{-1} g\right)^{\varphi}=a n^{-1} g$. This completes the proof since $\varphi$ fixes every element of the generating set $\left\{n, a n^{-1} g\right\} \cup A$ of $G$.

Finally, if $S$ has the fourth of the four possible forms, then $a n^{-1} g$ is the only vertex of $S \backslash B a$ that has only one outneighbour in $A$, so $\left(a n^{-1} g\right)^{\varphi}=a n^{-1} g$. Of the remaining three vertices of $S \backslash B a, a b_{1} n$ is the only one that has both $a^{2} b_{2} n^{2}$ and $a^{2} b_{3} n^{2}$ as outneighbours, so $\left(a b_{1} n\right)^{\varphi}=a b_{1} n$. This completes the proof since $\varphi$ fixes every element of the generating set $\left\{a n^{-1} g, a b_{1} n\right\} \cup A$ of $G$.

This shows that every group that arises in Proposition 4.1(c) that has order at least $2^{10}$ has an ORR.

\section{The groups in Theorem 2.3 (III)}

We discuss in some detail the work in [7] and establish some notation that we use for the rest of this section. Let $G, N, g$ and $n_{0}$ be as in Theorem 2.3 (iii). Thus $|G: N|=2, g^{2}=1, N=H \cup n_{0} H$ where $H:=\left\{n \in N \mid n^{g}=n^{-1}\right\}$ (note that $H$ is a set and not necessarily a group), $|H|=|N| / 2$ and $N$ has no automorphisms inverting more than half of its elements (according to Theorem 2.3. $N$ is a group described in the Hegarty and MacHale paper, and hence $N$ admits an automorphism inverting precisely half of its elements, namely conjugation via $g$, and no automorphisms inverting more than half of its elements). We observe that our assumption about the existence and properties of $n_{0}$ is not amongst the assumptions of Hegarty and MacHale, and we will be able to use this assumption to eliminate some of the groups in their classification.

The classification of Hegarty and MacHale is very satisfactory, but not very easy to use in our application. These groups fall into ten isoclinism classes and, for each class, the authors give a very explicit description of a stem group in the class. (We denote by $X^{\prime}$ the derived subgroup and by $\mathbf{Z}(X)$ the centre of the group $X$.) We recall that the groups $X$ and $Y$ are isoclinic if there exist two group automorphisms $\varphi: X^{\prime} \rightarrow Y^{\prime}$ and $\psi: X / \mathbf{Z}(X) \rightarrow Y / \mathbf{Z}(Y)$ with

$$
\left[x_{1} \mathbf{Z}(X), x_{2} \mathbf{Z}(X)\right]^{\varphi}=\left[\left(x_{1} \mathbf{Z}(X)\right)^{\psi},\left(x_{2} \mathbf{Z}(X)\right)^{\psi}\right], \quad \text { for every } x_{1}, x_{2} \in X .
$$

For instance, the dihedral group of order 8 and the quaternion group of order 8 are isoclinic. Being isoclinic is an equivalence relation coarser than the equivalence relation determined by the notion of group isomorphism. This means that finite groups are subdivided into isoclinism classes. Two groups in the same isoclinism 
class may not have the same order, for instance, $X$ and $X \times Z$ are isoclinic for every finite group $X$ and for every abelian group $Z$. It is well-known and also easy to prove that, for every group $X$, in the isoclinism class of $X$ there exists a group $Y$ with $\mathbf{Z}(Y) \leq Y^{\prime}$. A group satisfying $\mathbf{Z}(Y) \leq Y^{\prime}$ is said to be stem group. The definition of isoclinism yields that the stem groups are precisely the groups of smallest possible order within their isoclinism class. It is quite unfortunate that two stem groups may be isoclinic but not necessarily isomorphic: consider again the example of the dihedral and the quaternion group of order 8 .

We can now explain in some detail the work of Hegarty and MacHale. (Just for this paragraph, we say that $X$ is half-inverting, if $X$ has an automorphism inverting half of its elements and no automorphisms inverting more than half of its elements.) As a by-product of their main theorem, they prove that if $X$ and $Y$ are isoclinic and $X$ is half-inverting, then $Y$ is half-inverting. Moreover, Hegarty and MacHale prove that half-inverting groups fall into 10 distinct isoclinism classes. To describe these 10 isoclinism classes, Hegarty and MacHale exhibit 10 (non-isoclinic) stem groups: two of order 32, six of order 64 and two of order 128. We emphasise once again that this does not mean that there are only two stem groups of order 128 that are half-inverting. (A computation with the computer algebra system magma 3 shows that there are 5, 55 and 251 half-inverting stem groups of order 32, 64 and 128, respectively, up to isomorphism.)

Now that we have explained some details required for understanding the classification of Hegarty and MacHale, we can proceed to deal with the groups that arise in our context.

Proposition 5.1. Let $G$ be a group that arises in Theorem 2.3 (iii). Then $G$ admits an ORR except possibly if $N$ has an abelian subgroup $A$ with $|N: A|=4$, and $A$ is either isomorphic to $C_{4} \times C_{2}^{\ell}$ for some $\ell \leq 5$, or $A$ is elementary abelian of rank at most 7 .

Proof. By Theorem 2.3 (iii), $G$ is a 2-group with a normal subgroup $N,|G: N|=2$, and elements $g \in G \backslash N$ and $n_{0} \in N$ with $g^{2}=1$; the action of $g$ by conjugation on $N$ inverts precisely half of the elements of $N$; and $N=H \cup n_{0} H$, where $H$ := $\left\{n \in N \mid n^{g}=n^{-1}\right\}$, and $|H|=|N| / 2$.

From [7, Lemma 1] and its proof (see also the last paragraph of [7, page 132]), $N$ has an abelian subgroup $A$ with $|N: A|=4$ and with $a^{g}=a^{-1}$ for each $a \in A$. Some more information on the interaction between $A$ and $g$ and $N$ is available by reading the proof of Lemma 1 in [7] (again, see also the last paragraph of page 132 in [7]). Let $1, x_{2}, x_{3}, x_{4}$ be left coset representatives for $A$ in $N$; thus $N=A \cup x_{2} A \cup x_{3} A \cup x_{4} A$. According to Hegarty and MacHale, there are only two cases:

CASE I: $g$ inverts some element in each coset of $A$ in $N$;

CASE II: $g$ inverts some element in the cosets $A, x_{3} A$ and $x_{4} A$, and $g$ inverts no elements in the coset $x_{2} A$.

For the benefit of the reader, we have used the same subdivision into cases here, as the subdivision used in [7. Replacing $x_{2}, x_{3}, x_{4}$ by suitable coset representatives we may assume that $g$ inverts $x_{3}$ and $x_{4}$ and that $g$ also inverts $x_{2}$ in CASE I. Let $x \in\left\{x_{2}, x_{3}, x_{4}\right\}$ in CASE I and $x \in\left\{x_{3}, x_{4}\right\}$ in CASE II. Let $y \in H \cap x A$. Then, $y=$ $x a$ for some $a \in A$ and $a^{-1} x^{-1}=(x a)^{-1}=y^{-1}=y^{g}=(x a)^{g}=x^{g} a^{g}=x^{-1} a^{-1}$. 
This shows that $x a=a x$. Therefore

$$
H= \begin{cases}A \cup x_{2} \mathbf{C}_{A}\left(x_{2}\right) \cup x_{3} \mathbf{C}_{A}\left(x_{3}\right) \cup x_{4} \mathbf{C}_{A}\left(x_{4}\right) & \text { in CASE I, } \\ A \cup x_{3} \mathbf{C}_{A}\left(x_{3}\right) \cup x_{4} \mathbf{C}_{A}\left(x_{4}\right) & \text { in CASE II. }\end{cases}
$$

Theorem 2.3 (iii) gives us information about the element $n_{0}$ in addition to the structure that was studied by Hegarty and MacHale, and we have not used this in our analysis yet. We do so now. Recall that $N=H \cup n_{0} H$, and $H \cap n_{0} H=\emptyset$ because $H$ has cardinality $|N| / 2$.

Assume that CASE I holds. Now $n_{0} A \subseteq n_{0} H$ and hence $n_{0} H$ contains a whole left coset of $A$ in $N$. Since $H$ contains elements from each left coset of $A$ in $N$, we get $n_{0} H \cap H \neq \emptyset$, a contradiction. Therefore 2-groups in CASE I do not arise in Theorem 2.3 (iii).

For the rest of the proof, we assume that CASE II holds. From [7, line 11 from the bottom of page 134], we have $A \triangleleft N$ and $N / A$ is elementary abelian of order 4; therefore, $A \triangleleft G$ because $g$ also normalises $A$.

As

$$
2|A|=|N| / 2=|H|=|A|+\left|\mathbf{C}_{A}\left(x_{3}\right)\right|+\left|\mathbf{C}_{A}\left(x_{4}\right)\right|,
$$

we deduce $\left|A: \mathbf{C}_{A}\left(x_{3}\right)\right|=\left|A: \mathbf{C}_{A}\left(x_{4}\right)\right|=2$. Clearly, $n_{0} \in x_{2} A$, otherwise, arguing as in the previous paragraph, we get $n_{0} H \cap H \neq \emptyset$. Replacing $x_{2}$ if necessary, we may assume that $n_{0}=x_{2}$. Thus

$$
n_{0} H=x_{2} A \cup x_{2} x_{3} \mathbf{C}_{A}\left(x_{3}\right) \cup x_{2} x_{4} \mathbf{C}_{A}\left(x_{4}\right) .
$$

It is important to observe that since $A$ is normal in $N$ and $N / A$ is elementary abelian, we have $x_{2} x_{3} A=\left(x_{2} A\right)\left(x_{3} A\right)=x_{4} A$ and $x_{2} x_{4} A=\left(x_{2} A\right)\left(x_{4} A\right)=x_{3} A$; therefore $x_{2} x_{3} \mathbf{C}_{A}\left(x_{3}\right)$ is contained in the coset $x_{4} A$ and $x_{2} x_{4} \mathbf{C}_{A}\left(x_{4}\right)$ is contained in the coset $x_{3} A$. Now, the condition $N=H \cup n_{0} H$ yields $x_{3} A=x_{3} \mathbf{C}_{A}\left(x_{3}\right) \cup$ $x_{2} x_{4} \mathbf{C}_{A}\left(x_{4}\right)$. This implies that $A$ is the union of a coset of $\mathbf{C}_{A}\left(x_{4}\right)$ with a coset of $\mathbf{C}_{A}\left(x_{3}\right)$, and since each of these subgroups of $A$ has cardinality $|A| / 2$, this can happen only when $\mathbf{C}_{A}\left(x_{3}\right)=\mathbf{C}_{A}\left(x_{4}\right)$. Since $N=\left\langle A, x_{3}, x_{4}\right\rangle$, we have

$$
\mathbf{Z}(N)=\mathbf{C}_{A}\left(x_{3}\right) \cap \mathbf{C}_{A}\left(x_{4}\right)=\mathbf{C}_{A}\left(x_{4}\right)<A ;
$$

hence, $|N: \mathbf{Z}(N)|=8$. Finally we observe that $G / A$ is elementary abelian of order 8 because $g$ acts by conjugation inverting $x_{3}$ and $x_{4}$ and hence fixes the cosets $x_{3} A$ and $x_{4} A$.

Suppose now that $A$ is neither elementary abelian nor isomorphic to $C_{4} \times C_{2}^{\ell}$, for some $\ell \in \mathbb{N}$. From Lemma 2.7 there exists a subset $T$ of $A$ of cardinality at least 2 with $\operatorname{Cay}(A, T)$ an ORR and such that the subgraph induced by $\operatorname{Cay}(A, T)$ on $T$ is weakly connected.

We claim that $A=\mathbf{C}_{G}(A)$, that is, no element of $G \backslash A$ centralises $A$. Observe that

$$
G \backslash A=x_{3} A \cup x_{4} A \cup x_{3} x_{4} A \cup g x_{3} A \cup g x_{4} A \cup g x_{3} x_{4} A .
$$

From [7, Lemma 1], $N$ has no abelian subgroup of index less then four. Therefore no element in the cosets $x_{3} A, x_{4} A$ and $x_{3} x_{4} A$ centralises $A$ and, moreover,

$$
\mathbf{Z}(N)=\mathbf{C}_{A}\left(x_{3}\right)=\mathbf{C}_{A}\left(x_{4}\right)=\mathbf{C}_{A}\left(x_{3} x_{4}\right) .
$$

Observe now that $\mathbf{Z}(N)$ is not elementary abelian because $A$ has no elementary abelian subgroup of index 2 . Since $g$ acts by conjugation inverting each element of $\mathbf{Z}(N)$, we deduce that no element in the cosets $g A, g x_{3} A, g x_{4} A$ and $g x_{3} x_{4} A$ centralises $\mathbf{Z}(N) \leq A$. Thus our claim is proved. 
By Lemma 2.8, since $|G: A|=8$, there are generators $a_{1}, a_{2}, a_{3}$ for $G$ modulo $A$ none of which is an involution. Furthermore, since $G / A$ is elementary abelian and $A$ is abelian, $a_{i}^{2}$ centralises $A$ for every $i \in\{1,2,3\}$. From the previous paragraph, $a_{i} a_{j}$ does not centralise $A$, for every $i, j \in\{1,2,3\}$ with $i \neq j$. In particular, the second hypothesis of Lemma 2.8 holds, and we deduce that $G$ admits an ORR.

Suppose now that $A$ is either elementary abelian or isomorphic to $C_{4} \times C_{2}^{\ell}$ for some $\ell \in \mathbb{N}$. We deal with the two possible structures for $A$ individually.

Suppose first that $A$ is isomorphic to $C_{4} \times C_{2}^{\ell}$. By ignoring the exceptions from our statement, we may assume that $\ell \geq 6$. Let $a_{0} \in A \backslash \mathbf{Z}(N)$ with $o\left(a_{0}\right)=4$ (observe that this is possible because $\mathbf{Z}(N)<N$ cannot contain all the elements of order 4 of $A$ ). Let $B$ be an elementary abelian subgroup of rank $\ell$ in $A$ that does not include the non-identity square element of $A$, and let $T$ be the Imrich generating set for $B$ with respect to some generating tuple. Let

$$
X:=\left\{g x_{3} a_{0}, g x_{3} a_{0} b, g x_{3} a_{0} b^{\prime}, g x_{3} a_{0} b^{\prime \prime}\right\},
$$

where $b, b^{\prime}$, and $b^{\prime \prime}$ are chosen from $B$ so that $\left\langle b, b^{\prime}, b^{\prime \prime}\right\rangle$ has order 8 and none of the pairwise products from $\left\{1, b, b^{\prime}, b^{\prime \prime}\right\}$ is $\left(a_{0}^{-1}\right)^{x_{3}} a_{0}$ : the rank of $B$ is easily large enough that such choices are possible. Let

$$
Y:=\left\{g x_{4} a_{0}, g x_{4} a_{0} c, g x_{4} a_{0} c^{\prime}\right\}
$$

where $c$ and $c^{\prime}$ are chosen from $B$ so that $\left\langle c, c^{\prime}\right\rangle$ has order 4 and none of the pairwise products from $\left\{1, c, c^{\prime}\right\}$ is $\left(a_{0}^{-1}\right)^{x_{4}} a_{0}$ (again, this is possible because $B$ has rank at least 6$)$. Let

$$
S:=\left[\left(B a_{0} \backslash T a_{0}\right) \backslash\left\{a_{0}\right\}\right] \cup X \cup Y \cup\left\{g x_{3} x_{4}\right\} .
$$

The elements of $S \cap B a_{0}$ have order 4 and none is the inverse of another since $a_{0}^{2} \notin B$. The pairwise products of the elements of $S$ that are not in $A$ cannot yield the identity. Furthermore, observe that, for every $z, z^{\prime} \in\left\{1, b, b^{\prime}, b^{\prime \prime}\right\}$, we have

$$
\left(g x_{3} a_{0} z\right)\left(g x_{3} a_{0} z^{\prime}\right)=\left(a_{0}^{-1}\right)^{x_{3}} a_{0} z z^{\prime} \neq 1
$$

by our choice of $b, b^{\prime}$ and $b^{\prime \prime}$ and the fact that $a_{0} \notin \mathbf{Z}(N)=\mathbf{C}_{A}\left(x_{3}\right)$. Similarly, for every $z, z^{\prime} \in\left\{1, c, c^{\prime}\right\}$, we have

$$
\left(g x_{4} a_{0} z\right)\left(g x_{4} a_{0} z^{\prime}\right)=\left(a_{0}^{-1}\right)^{x_{4}} a_{0} z z^{\prime} \neq 1
$$

by our choice of $c$ and $c^{\prime}$ and the fact that $a_{0} \notin \mathbf{Z}(N)=\mathbf{C}_{A}\left(x_{4}\right)$. Finally,

$$
\left(g x_{3} x_{4}\right)^{2}=g x_{3} x_{4} g x_{3} x_{4}=x_{3}^{g} x_{4}^{g} x_{3} x_{4}=x_{3}^{-1} x_{4}^{-1} x_{3} x_{4} \neq 1,
$$

where in the third equality we have used $x_{3}, x_{4} \in H=\left\{n \in N \mid n^{g}=n^{-1}\right\}$, and in the last inequality we used the fact that $x_{3}$ and $x_{4}$ do not commute because $x_{3} x_{4} \notin H$ and

$$
x_{4}^{-1} x_{3}^{-1}=\left(x_{3} x_{4}\right)^{-1} \neq\left(x_{3} x_{4}\right)^{g}=x_{3}^{g} x_{4}^{g}=x_{3}^{-1} x_{4}^{-1} .
$$

This proves that $S \cap S^{-1}=\emptyset$ and $\operatorname{Cay}(G, S)$ is an oriented graph.

By Proposition 3.3. any automorphism $\varphi \in \operatorname{Aut}(\operatorname{Cay}(G, S))_{1}$ fixes $B$ and $a_{0}$ pointwise, so fixes $\left\langle a_{0}, B\right\rangle=A$ pointwise.

Observe that, for any of the elements of $X$, its outneighbours via elements of $X \cup Y \cup\left\{g x_{3} x_{4}\right\}$ have four outneighbours in $A$ and four not in $A$; the elements of $Y$ each have three outneighbours via $X \cup Y \cup\left\{g x_{3} x_{4}\right\}$ in $A$ and five not in $A$; and $g x_{3} x_{4}$ has only one outneighbour via $X \cup Y \cup\left\{g x_{3} x_{4}\right\}$ that is in $A$. Thus $X^{\varphi}=X, Y^{\varphi}=Y$, and $\left(g x_{3} x_{4}\right)^{\varphi}=g x_{3} x_{4}$, for every $\varphi \in \operatorname{Aut}(\operatorname{Cay}(G, S))_{1}$. Furthermore, in $X$, since $\left\langle b, b^{\prime}, b^{\prime \prime}\right\rangle$ has order $8, g x_{3} a_{0}$ is the only vertex that does not have either $\left(a_{0}^{-1}\right)^{x_{3}} a_{0} b b^{\prime}$ 
or $\left(a_{0}^{-1}\right)_{3}^{x} a_{0} b b^{\prime \prime}$ as an outneighbour. Therefore $g x_{3} a_{0}$ is fixed by $\operatorname{Aut}(\operatorname{Cay}(G, S))_{1}$. Similarly, in $Y$, since $\left\langle c, c^{\prime}\right\rangle$ has order $4, g x_{4} a_{0}$ is the only vertex that does not have $\left(a_{0}^{-1}\right)^{x_{4}} a_{0} c c^{\prime}$ as an outneighbour. Therefore $g x_{4} a_{0}$ is fixed by $\operatorname{Aut}(\operatorname{Cay}(G, S))_{1}$. Thus $\operatorname{Aut}(\operatorname{Cay}(G, S))_{1}$ fixes the generating set $\left\{g x_{3} x_{4}, g x_{3} a_{0}, g x_{4} a_{0}\right\} \cup A$ for $G$ pointwise, so by Lemma 2.6. $\operatorname{Aut}(\operatorname{Cay}(G, S))_{1}=1$ and $\operatorname{Cay}(G, S)$ is an ORR.

Suppose now that $A$ is elementary abelian, that is, $A \cong C_{2}^{\ell}$ for some $\ell \in \mathbb{N}$. By ignoring the exceptions from our statement, we may assume that $\ell \geq 8$. As $x_{3}$ and $x_{4}$ do not commute, the commutator $d:=x_{4}^{-1} x_{3}^{-1} x_{4} x_{3} \neq 1$. Recall that $N / \mathbf{Z}(N)$ is elementary abelian and hence the commutator subgroup $N^{\prime}$ is contained in $\mathbf{Z}(N)$. Thus $d \in \mathbf{Z}(N)$. Let $d, b_{1}, \ldots, b_{\ell-2}$ be an irredundant generating set for $\mathbf{Z}(N) \cong C_{2}^{\ell-1}$, and let $B:=\left\langle b_{1}, \ldots, b_{\ell-2}\right\rangle$. So $B$ is elementary abelian of rank $\ell-2 \geq 6$. Let $T$ be the Imrich generating set for $B$ with respect to some generating tuple.

Let $a_{0} \in A \backslash \mathbf{Z}(N)$ and observe that $\left\langle\mathbf{Z}(N), g, x_{3} a_{0}, x_{4} a_{0}\right\rangle$ is a subgroup of $G$ having index 2. Since $G$ is not generalised dihedral, there exists $v \in G \backslash$ $\left\langle\mathbf{Z}(N), g, x_{3} a_{0}, x_{4} a_{0}\right\rangle$ with $o(v)>2$. Let

$$
X:=\left\{g x_{3} a_{0}, g x_{3} a_{0} b, g x_{3} a_{0} b^{\prime}, g x_{3} a_{0} b^{\prime \prime}\right\},
$$

where $b, b^{\prime}$, and $b^{\prime \prime}$ are chosen from $B$ so that $\left\langle b, b^{\prime}, b^{\prime \prime}\right\rangle$ has order 8 and none of the pairwise products from $\left\{1, b, b^{\prime}, b^{\prime \prime}\right\}$ is $\left(a_{0}^{-1}\right)^{x_{3}} a_{0}$ : the rank of $B$ is easily large enough that such choices are possible. Let

$$
Y:=\left\{g x_{4} a_{0}, g x_{4} a_{0} c, g x_{4} a_{0} c^{\prime}\right\},
$$

where $c$ and $c^{\prime}$ are chosen from $B$ so that $\left\langle c, c^{\prime}\right\rangle$ has order 4 and none of the pairwise products from $\left\{1, c, c^{\prime}\right\}$ is $\left(a_{0}^{-1}\right)^{x_{4}} a_{0}$ (again, this is possible because $B$ has rank at least 6$)$. Let

$$
S:=g x_{3} x_{4}[(B \backslash T) \backslash\{1\}] \cup X \cup Y \cup\{v\} \cup\left\{g x_{3} x_{4} d\right\} .
$$

For any $z, z^{\prime} \in B$, we have

$$
\left(g x_{3} x_{4} z\right)\left(g x_{3} x_{4} z^{\prime}\right)=x_{3}^{-1} x_{4}^{-1} x_{3} x_{4} z z^{\prime}=d z z^{\prime} \neq 1,
$$

because $d \notin B$. The argument that no two elements of $X$ or $Y$ are inverses of one another is exactly as in the previous case. Notice also that $v$ is not in $\langle S \backslash\{v\}\rangle$, so $o(v)>2$ implies that $v^{-1} \notin S$. Now, observe that, for any $b \in B \backslash\{1\}$, we have

$$
g x_{3} x_{4} b g x_{3} x_{4} d=d^{x_{4}} b d=d b d=b \neq 1
$$

since $d \in \mathbf{Z}(N)$ which is elementary abelian. Finally, $\left(g x_{3} x_{4} d\right)^{2}=d \neq 1$. Thus $S \cap S^{-1}=\emptyset$ and $\operatorname{Cay}(G, S)$ is an oriented Cayley graph.

Observe that $\left(g x_{3} x_{4}\right)^{2}=d$ implies $o\left(g x_{3} x_{4}\right)=4$, and since $\mathbf{Z}(N)$ is elementary abelian we have $B<\mathbf{Z}(N)=\mathbf{Z}(G)$, so $g x_{3} x_{4}$ centralises $B$. Thus Proposition 3.3 applies to show that $\operatorname{Aut}(\operatorname{Cay}(G, S))_{1}$ fixes $B$ and $g x_{3} x_{4}$ pointwise. Thus the cosets of $B$ are blocks of imprimitivity for $\operatorname{Aut}(\operatorname{Cay}(G, S))_{1}$. Since the four vertices of $X$ lie in one coset of $B$, the three vertices of $Y$ lie in a different coset, and the vertices $v$ and $g x_{3} x_{4} d$ each lie in a different coset of $B$, we have $X^{\varphi}=X$ and $Y^{\varphi}=Y$, for every $\varphi \in \operatorname{Aut}(\operatorname{Cay}(G, S))_{1}$. The same argument as in the previous case again shows that $g x_{3} a_{0}$ and $g x_{4} a_{0}$ are fixed by $\operatorname{Aut}(\operatorname{Cay}(G, S))_{1}$.

Let $\varphi \in \operatorname{Aut}(\operatorname{Cay}(G, S))_{1}$. Since $d \in \mathbf{Z}(N)$ has order 2 , we see that $g x_{3} x_{4} d$ has $2^{\ell}-2 \ell-2$ outneighbours in $B$, but $v$ has at most one outneighbour in $B$. Thus $v^{\varphi}=v$ and $\left(g x_{3} x_{4} d\right)^{\varphi}=g x_{3} x_{4} d$. We now see that $\varphi$ fixes every point of the 
generating set $\left\{g x_{3} a_{0}, g x_{4} a_{0}, v, g x_{3} x_{4}, g x_{3} x_{4} d\right\} \cup B$ for $G$. Since $\varphi$ was arbitrary, Lemma 2.6 gives that $\operatorname{Cay}(G, S)$ is an ORR.

\section{Proof of Theorem 1.2}

We are now ready to prove our main theorem, Theorem 1.2

Proof of Theorem 1.2. If $|G|=2$, then Cay $(G, \emptyset)$ is an ORR for $G$. We henceforth assume that $G$ is not generalised dihedral.

By Theorem 2.3. $G$ admits an ORR unless $G$ is as in Theorem 2.3 (ii), Theorem 2.3 (iii), or $G \cong Q_{8}, C_{3}^{2}$, or $C_{3} \times C_{2}^{3}$. The final three possibilities are in our list of exceptions in the statement of Theorem 1.2. Suppose now that $G$ is as in Theorem 2.3 (ii). By Proposition 4.1, either $G$ admits an ORR, or one of the following possibilities holds:

(1) $\ell$ and $\kappa$ are non-negative integers, $V$ is an elementary abelian 2-group of rank $2 \ell+\kappa$ with generating set $\left\{v_{1}, w_{1}, \ldots, v_{\ell}, w_{\ell}, e_{1}, \ldots, e_{\kappa}\right\}$, and $G=$ $\langle V, x\rangle$, where $v_{i}^{x}=w_{i}, w_{i}^{x}=v_{i}$ for $i \in\{1, \ldots, \ell\}$ and $e_{i}^{x}=e_{i}$ for $i \in$ $\{1, \ldots, \kappa\}$; and

(i) $x^{2}=1$; or

(ii) $\kappa \geq 1$ and $x^{2}=e_{1}$; or

(2) $\langle A, g\rangle$ is isomorphic to $D_{4} \times C_{2}^{\ell}$ for some $\ell \in \mathbb{N}$.

If (1)(i) holds, then by Lemma 4.2 we have $\ell \geq 2$. Now by Lemma 4.3, $G$ admits an ORR as long as $2 \ell+\kappa \geq 8$; equivalently, as long as $|G| \geq 2^{9}$. If (1)(ii) holds, then by Lemma 4.4. $G$ admits an ORR as long as $2 \ell+\kappa \geq 7$; equivalently, as long as $|G| \geq 2^{8}$. If (2) holds, then by Lemma 4.8, $G$ admits an ORR as long as $\ell \geq 6$; equivalently, as long as $|G| \geq 2^{10}$. On the other hand, if $G$ is as in Theorem 2.3 (iii), then by Proposition 5.1, $G$ admits an ORR when $|G| \geq 2^{11}$.

It remains to check 2 -groups of order at most $2^{9}$ that are not generalised dihedral and that satisfy Theorem 2.3 (ii) and the 2 -groups of order at most $2^{10}$ that satisfy Proposition 5.1. These were all checked with the aid of magma, and the only groups that do not admit an ORR are those listed.

\section{REFERENCES}

[1] BibliographyL. Babai, 'Finite digraphs with given regular automorphism groups', Periodica Mathematica Hungarica 11 (1980), 257-270.

[2] BibliographyL. Babai and W. Imrich, 'Tournaments with given regular group', Aequationes Mathematicae 19 (1979), 232-244.

[3] BibliographyW. Bosma, J. Cannon and C. Playoust, 'The Magma algebra system. I. The user language', J. Symbolic Comput. 24 (1997), 235-265.

[4] BibliographyM. Conder, T. Tucker, and M. Watkins, 'Graphical Frobenius Representations with even complements', https://www.math.auckland.ac.nz/ dleemans/SCDO2016/SCDO-TALKS/Monday/Tucker.pdf

[5] BibliographyE. Dobson, P. Spiga, and G. Verret, 'Cayley graphs on abelian groups', Combinatorica 36 (2016), 371-393.

[6] BibliographyC. D. Godsil, 'GRRs for nonsolvable groups', Algebraic Methods in Graph Theory, (Szeged, 1978), 221-239, Colloq. Math. Soc. János Bolyai 25, North-Holland, Amsterdam-New York, 1981.

[7] BibliographyP. Hegarty and D. MacHale, 'Two-groups in which an automorphism inverts precisely half of the elements', Bull. London Math. Soc. 30 (1998), 129-135.

[8] BibliographyD. Hetzel, 'Über reguläre graphische Darstellung von auflösbaren Gruppen', Technische Universität, Berlin, 1976. 
[9] BibliographyW. Imrich, 'Graphs with transitive abelian automorphism group', Combinat. Theory (Proc. Colloq., Balatonfüred, 1969), Budapest, 1970, 651-656.

[10] BibliographyL. A. Nowitz and M. E. Watkins, 'Graphical regular representations of nonabelian groups. I', Canad. J. Math. 24 (1972), 993-1008.

[11] BibliographyJ. Morris, P. Spiga and G. Verret, 'Automorphisms of Cayley graphs on generalised dicyclic groups', European Journal of Combinatorics 43 (2015), 68-81.

[12] BibliographyJ. Morris and P. Spiga, 'Every Finite Non-Solvable Group admits an Oriented Regular Representation', Journal of Combinatorial Theory Series B, to appear.

[13] BibliographyP. Spiga, 'Finite groups admitting an oriented regular representation', Journal of Combinatorial Theory Series A, to appear.

[14] BibliographyP. Spiga, 'Cubic graphical regular representations of finite non-abelian simple groups', submitted.

[15] BibliographyS. J. Xu, X. G. Fang, J. Wang and M. Xu, 'On cubic s-arc transitive Cayley graphs of finite simple groups', European J. Combin. 26 (2005), 133-143.

Joy Morris, Department of Mathematics and Computer Science, University of LethBridge, Lethbridge, AB. T1K 3M4. CAnada

E-mail address: joy.morris@uleth.ca

Pablo Spiga, Dipartimento di Matematica e Applicazioni, University of Milano-Bicocca, Via Cozzi 55, 20125 Milano, Italy

E-mail address: pablo.spiga@unimib.it 